\title{
Thermoviscoplastic behaviors of anisotropic shape memory elastomeric composites for cold programmed non-affine shape change
}

\author{
Yiqi Mao ${ }^{a}$, Jaimee M. Robertson ${ }^{b}$, Xiaoming Mu ${ }^{a}$, Patrick T. Mather ${ }^{b}$, H. Jerry Qi ${ }^{a(*)}$ \\ ${ }^{\mathrm{a}}$ The George Woodruff School of Mechanical Engineering, Georgia Institute of Technology, Atlanta, GA \\ 30332, USA \\ ${ }^{\mathrm{b}}$ Department of Biomedical and Chemical Engineering, Syracuse University, Syracuse, NY 13244, USA
}

Corresponding author. Tel.: 404-385-2457; E-mail address: qih@me.gatech.edu 
Abstract : Shape memory polymers (SMPs) can fix a temporary shape and recover their permanent shape upon activation by an external stimulus. Most SMPs require programming at above their transition temperatures, normally well above the room temperature. In addition, most SMPs are programmed into shapes that are affine to the high temperature deformation. Recently, a cold-programmed anisotropic shape memory elastomeric composite was developed and showed interesting low temperature stretching induced shape memory behavior. There, simple, uniaxial stretching at low temperature transformed the composites into curled temporary shapes upon unloading. The exact geometry of the curled state depended on the microstructure of the composite, and the curled shape showed no affinity to the deformed shape. Heating the sample recovered the sample back to its original shape. This new composite consisted of an elastomeric matrix reinforced by aligned amorphous polymer fibers. By utilizing the plastic-like behavior of the amorphous polymer phase at low temperatures, a temporary shape could be fixed upon unloading since the induced plastic-like strain resists the recovery of the elastomer matrix. After heating to a high temperature, the permanent shape was recovered when the amorphous polymer softened and the elastomer matrix contracted. To set a theoretical foundation for capturing the cold-programmed shape memory effects and the dramatic non-affine shape change of this composite, a 3D anisotropic thermoviscoelastic constitutive model is developed in this paper. In this model, the matrix is modeled as a hyperelastic solid, and the amorphous phase of the fibrous mat is considered as a nonlinear thermoviscoplastic solid, whose viscous flow resistance is sensitive to both temperature and stress. The plastic-deformation like behavior demonstrated in the fiber is treated as a nonlinear viscoplasticity with extremely high viscosity or long relaxation time at zero-stress state at low temperature. The anisotropic viscoplastic property of the fibrous mat is captured by an isotropic fibrous network superimposed with an oriented fibrous network. The material parameters in the model are identified from the experiments on the fibrous mat and on the composites, respectively. The cold-programmed shape memory behaviors of the composite are predicted by simulations and compared with experiments without further adjusting the material parameters. Good agreement is observed, indicating the ability of the present model to 
capture the anisotropic viscoplastic and shape memory behaviors. By using the developed constitutive model, effects of loading rate and fiber volume fraction on cold programmed shape memory behavior are discussed. Furthermore, the constitutive relation is applied to a mechanical model to study the cold-programmed curling of the laminates.

Keyword: Shape memory polymers; soft materials; active composites; constitutive model; thermoviscoplasticity. 


\section{Introduction}

Shape memory polymers (SMPs) have the ability to "memorize" a permanent shape. After being fixed to a temporary shape, an SMP can recover its original permanent shape upon activation by an external stimulus (Hu and Zhuo, 2010; Hu et al., 2012; Lendlein and Kelch, 2002; Leng et al., 2011; Liu et al., 2007; Lu et al., 2013; Meng and Li, 2013; Yang et al., 2014). In a typical thermally triggered SMP, which is the most common and intensively researched, the SMP is first heated to a high temperature above a transition temperature and deformed into a new shape; it is then cooled to below the transition temperature; upon removal of the external load and constraints, the SMP maintains the programmed shape. Upon heating to a temperature above the transition temperature, it recovers its original shape. The typical transition temperatures used for achieving shape memory (SM) effects include those for glass transition or crystal-melt transition (Ge et al., 2013b; Nguyen, 2013). Most applications require that the materials have the programmed shape and the permanent shape at room temperature, which means that the transition temperature should be well above the room temperature; thus, the programming temperature is typically in the range of $50^{\circ} \mathrm{C}-120^{\circ} \mathrm{C}$, depending on the material systems. This leads to two potential issues. First, shape programming at high temperatures is not easy, as heating is typically done in a thermal chamber, in which manipulating the sample is not a convenient task if one would like to achieve a complicated shape. Second, heating requires more energy, which in general is undesirable. It is also worth mentioning that despite the extensive number of studies on the SM phenomena, the past studies focused mainly on isotropic behaviors, and comparatively, very little attention has been given to the construction of SMP systems that exhibit anisotropic behavior while such anisotropy is very common in natural materials(Rodriguez et al., 2013). Moreover, most SM effects in SMPs so far are limited to affine programming; i.e., the temporary shape is an affine transformation of the deformed shape at high temperature. Cold programming $\mathrm{SM}$ effects have been explored in $(\mathrm{Li}$ and $\mathrm{Xu}, 2011 ; \mathrm{Li}$ and Shojaei, 2012; Ping et al., 2005; Shojaei et al., 2013). For example, Li and Xu (2011) have developed an isotropic viscoelastic model which was demonstrated well in capturing isotropic 
cold compression shape memory effect. Li and Saojaei[13] and Shojaei et al[14] studied the evolution of microstructures in these fibers and the feasibility of applying them to self-healing materials. An anisotropic shape memory effect has been discussed under high temperature programming in (Ge et al., 2013a). Very few non-affine transformations have been seen in the past, and systematic studies of combined cold, non-affine programming and anisotropic effects are lacking.

Recently, Rodriguez et al. (2013) reported an anisotropic shape-memory elastomeric composite (ASMEC), which was constructed by infiltrating Sylgard-184 silicone resin into the the oriented electrospun fiber mat of poly(vinyl acetate) (PVAc). The silicone was subsequently cured, forming a rubbery matrix. There, PVAc served for shape memory fixing through its glass transition, and Sylgard-184 served as the soft matrix assisting in the SM recovery. Because the fibers had a dominant orientation, the shape fixing ability depended on the fiber orientation, and thus, the composite exhibited anisotropic SM effects, as was shown in a recent study(Ge et al., 2013a). In addition, it was demonstrated that the composites could be deformed at low temperature when the fibers were still in a glassy state. The excessive deformation of the fiber mat led to plastic-like deformation, which prevented the matrix material from recovering upon removal of the external load, allowing cold programming of SMPs. Moreover, when ASMECs were laminated into a multilayer structure, the cold programming could introduce non-affine shape change. For example, a simple stretching at low temperature could change a strip of laminate into a helix due to the non-uniform shape fixing resulting from fiber anisotropy. When heating to a higher temperature above the glass transition temperature of PVAc, the PVAc fibers became a viscous melt, thus permitting the recovery of the matrix material through its rubber elasticity. Three apparent advantages of this new class of SM composites are important for potential future applications. First, a continuously varying fixity is possible as the loading direction changes, which can be taken as a new design variable. Second, the cold programming can be done at the room temperature, which is very convenient and energy saving. Third, the ability to achieve non-affine shape programming further increases the design flexibility, as 
deforming a sample into a complicated shape can be non-trivial whilst non-affine shape change can provide an easy solution. These advantages of ASMECs widen the application prospects in medical devices and actuators/sensors. For example, ASMECs can find application in mimicking the skin membrane of a bat wing, a distinctive soft anisotropic fibrous network. In addition, a similar mechanical anisotropy could be useful for micro air vehicle systems featuring flapping wings, with the ASMEC helping to improve the adaptability, agility, and speed in unmanned aerodynamic vehicles(Rodriguez et al., 2013).

To establish a theoretical foundation providing a comprehensive understanding of thermomechanical behaviors for design with ASMEC materials, a constitutive model is needed. Since the cold-programmed ASMEC utilizes the plastic-like deformation behavior of the fibrous mat to obtain anisotropic shape fixity at low temperatures and the programmed shape can recover upon heating, the theoretical model should be able to capture the plastic-like and anisotropic behaviors of the composite and their dependence on temperature. In the past, several works have been carried out on finite deformation anisotropic constitutive models for fiber reinforced composites. Based on how strain energy functions are constructed, there are roughly two approaches. One uses the strain components directly and usually considers the material orthotropic. This group includes models from Kroon et al(2010) for the growth and remodeling of biological soft tissues, Ehret et al. (2011) for the passive and active mechanical behavior of skeletal muscle, Itskov et al. (2006) for anisotropic hyperelastic behavior of soft biological tissues, and Siliberstein et al. (2012) for the elasto-plastic behaviors of an electrospun non-woven fibrous mat. Another common approach is to use the invariants of the deformation tensor and fiber directions to construct strain energy functions. For example, Guo et al. (Guo et al., 2006, 2007) developed models for the hyperelastic behaviors of fiber reinforced composite by explicitly considering the fiber-matrix interactions and showed very good predictability. Such an approach is very successful in capturing the anisotropic hyperelastic behavior of composites or biological tissues(Holzapfel et al., 2000; Kao et al., 2011). For the modeling of time-dependent, anisotropic viscous behavior, Nguyen et al. (2008b) presented a constitutive model for the finite 
deformation, anisotropic, nonlinear viscoelastic behavior of the corneal stroma. The model formulated the elastic and viscous response of the stroma as an average of the elastic and viscous responses of the individual lamellae and was weighted by a probability density function of the preferred in-plane lamellar orientations. Holzapfel and Gasser (2001) developed a viscoelastic model for the fully three-dimensional stress and deformation response of fiber-reinforced composites in which soft matrix materials were reinforced by two families of fibers.

The goals of this paper are to establish a constitutive model for the ASMEC at the lamina level and to apply the lamina model to study the anisotropic shape memory effects as well as the active shape changing of laminates. At the lamina level modeling, an anisotropic thermoviscoplastic model for the ASMEC is developed. Here, the fibrous mat is modeled as an anisotropic viscoplastic solid with distinct fiber orientations, and the matrix is treated as an hyperelastic elastomer. The free energy functions for the fibrous mat and matrix are given in terms of invariants of the deformation tensor and fiber direction. By calibrating model parameters from experiments on the fiber reinforced composite, numerical examples are carried out for comparison purpose. The established constitutive relations with the fitted material parameters are applied to simulate the mechanically fixed SM behavior of ASMECs. The model is then applied to study cold programming for non-affine shape change of ASEMC laminate. The current work is in many ways different from the constitutive model for shape memory elastomeric composites (SMEC) previously reported by our group(Ge et al., 2012). There, only an isotropic scenario was considered; in addition, the fibrous mat under consideration went through a crystal-melt transition and shape fixing was done in a typical high temperature programing shape memory cycle. The layout of this work is arranged as the following: Section 2 describes the materials and the experimental processes and results. Section 3 focuses on establishing the 3D anisotropic viscoplastic constitutive model, and the mechanically fixed shape memory model. Section 4 presents the numerical simulations and results.

\section{Experimental Results}

\subsection{Materials}


The ASMEC in this study was composed of two polymers: a crosslinked PDMS (poly (dimethylsiloxane), Sylgard) and PVAc (poly(vinyl acetate)). The PVAc served as the fibrous mat for shape fixing and PDMS served as the soft matrix assisting the shape recovery. Details regarding the fabrication process and the resulting morphology of the anisotropic fibrous mat and ASMEC can be found in (Rodriguez et al., 2013). Fig.1 shows micrographs of the fibrous mat after electrospinning and of the composite. From Fig.1a, it can be seen that, overall, the PVAc fibers are well aligned, although there are some fibers deviating from the main direction. The top surface of the composite is homogeneous and smooth (Fig 1b). The cross-sections (Fig. 1c \&d) show a homogeneous mixture of the two materials.

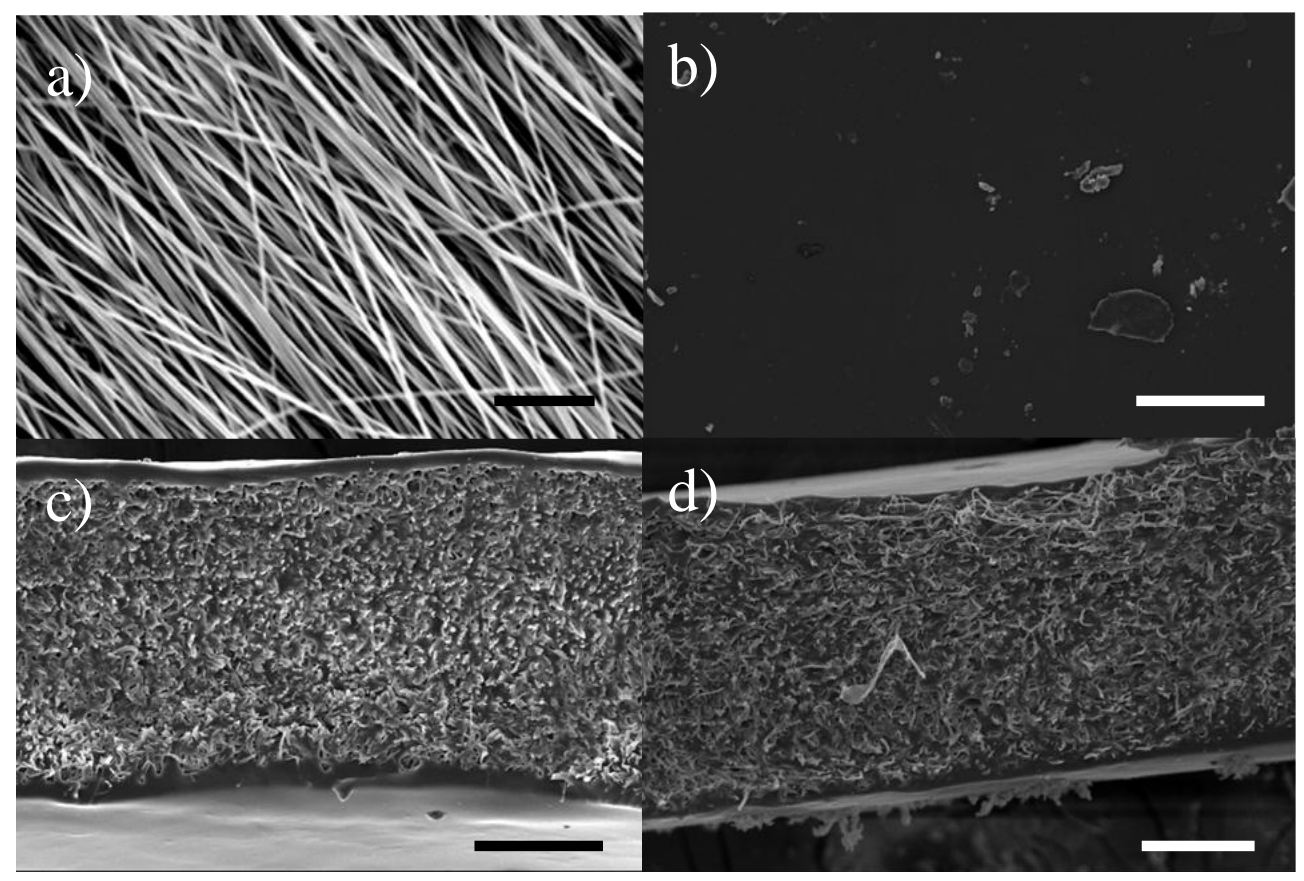

Figure 1. SEM micrographs of (a) PVAc fibrous mat; (b) surface of single ASMEC lamina; (c) lateral cross-section of the lamina; (d) longitudinal cross-section of the lamina. The scale bars are $20 \mu \mathrm{m}, 50 \mu \mathrm{m}, 50 \mu \mathrm{m}$, and $50 \mu \mathrm{m}$, respectively.

To fabricate the ASMEC laminate, squares (30 $\mathrm{mm}$ in length) were cut from the anisotropic single layer composite at various fiber angles $\left(\theta=0^{\circ}, 22.5^{\circ}, 45^{\circ}, 67.5^{\circ}\right.$ and $\left.90^{\circ}\right)$. Here, the angles were measured between the direction of the fibers and the cutting direction in the PVAc fiber mat. The single layer ASMECs had typical thicknesses of $0.3 \mathrm{~mm}$ and fiber weight fractions of 0.15 . 
Two plies were stacked and adhered with a layer of Sylgard, the thickness of which was measured to be less than $16 \%$ of the total laminate thickness. Rectangular strips $(30(\mathrm{l}) \times 2.5(\mathrm{w}) \times 0.7(\mathrm{t}) \mathrm{mm}$ average dimensions) were cut from the laminated ASMECs for testing. The prepared bilayers had fiber angles of $0^{\circ} / 0^{\circ}, 22.5^{\circ} / 0^{\circ}, 45^{\circ} / 0^{\circ}, 67.5^{\circ} / 0^{\circ}$, and $90 \% 0^{\circ}$ (top fiber orientation/bottom fiber orientation). Since each prepared laminated ASMEC had a bottom layer with $0^{\circ}$ fiber orientation, we will hereafter use the term " $\bar{\theta}$ " which refers to the difference in the fiber angle between the two layers $\left(\bar{\theta}=0^{\circ}, 22.5^{\circ}, 45^{\circ}, 67.5^{\circ}\right.$, and $\left.90^{\circ}\right)$. The laminated ASMECs were put in a vacuum oven for 1 hour to remove air bubbles from the Sylgard layer in between the two plies. After removing, the laminated ASMECs were cured at $30^{\circ} \mathrm{C}$ for 48 hour and then post cured at $60{ }^{\circ} \mathrm{C}$ for 16 hour.

\subsection{Thermal Properties}

Thermal properties of samples were characterized using Differential Scanning Calorimetry (DSC, Q200, TA Instruments, New Castle, DE, USA). Samples were heated from $-60{ }^{\circ} \mathrm{C}$ to $160{ }^{\circ} \mathrm{C}$ to remove the thermal history, cooled to $-60{ }^{\circ} \mathrm{C}$, and finally heated to $160{ }^{\circ} \mathrm{C}$ at the rate of $10{ }^{\circ} \mathrm{C}$ $\min ^{-1}$ to determine the glass transition temperatures, $T_{g}$. The results from the second cooling and heating cycle were reported. DSC was performed on PVAc fibrous mats and a single ASMEC lamina, respectively. The measured $T_{g} \mathrm{~s}$ are $42{ }^{\circ} \mathrm{C}$ and $46^{\circ} \mathrm{C}$ for the PVAc fibrous mat and the single ASMEC lamina (shown in Fig.2), respectively. As expected, no melting transition was observed for PVAc, an amorphous polymer. The $T_{g}$ of Sylgard was previously determined as -114 ${ }^{\circ} \mathrm{C}$ (Luo and Mather, 2009), and therefore, was not tested in our DSC experiments.

The thermal strains of the PVAc fiber mat and the single layer ASMEC were measured using a dynamic mechanical analyzer (DMA, Model Q800, TA Instruments, New Castle, DE, USA) in static, controlled stress mode. Strip samples with size of $12 \mathrm{~mm} \times 3.5 \mathrm{~mm} \times 0.4 \mathrm{~mm}$ were cut from the neat PVAc mat and the single ASMEC lamina along $0^{\circ}$ and $90^{\circ}$ direction, respectively. A small stretching force $\left(1 \times 10^{-4} \mathrm{~N}\right)$ was applied to ensure the straightness of the sample. The temperature was decreased from $70{ }^{\circ} \mathrm{C}$ to $10^{\circ} \mathrm{C}$ at a rate of $2{ }^{\circ} \mathrm{C} / \mathrm{min}$, and the thermal strains were recorded. From the Fig.3, it can be found that the PVAc material first 
contracted with a coefficient of thermal expansion (CTE) of $2.21 \times 10^{-4} /{ }^{\circ} \mathrm{C}$. When temperature reached around $40{ }^{\circ} \mathrm{C}$, there was a decrease in the CTE due to the glass transition. The CTE of PVAc is around $1.02 \times 10^{-4} /{ }^{\circ} \mathrm{C}$ when the temperature is below $40^{\circ} \mathrm{C}$. The anisotropic thermal expansion behavior of the ASMEC can also be observed from Fig.3. When the temperature is above the $T_{g}\left(46^{\circ} \mathrm{C}\right)$ of the ASMEC, the CTEs in the directions along and transverse to the fiber direction are $2.91 \times 10^{-4} /{ }^{\circ} \mathrm{C}$ and $3.01 \times 10^{-4} /{ }^{\circ} \mathrm{C}$, respectively. When the temperature is below the glass transition temperature, the anisotropic thermal behavior of the ASMEC is more obvious with CTEs of $1.23 \times 10^{-4} /{ }^{\circ} \mathrm{C}$ and $1.52 \times 10^{-4} /{ }^{\circ} \mathrm{C}$ in the fiber and transverse directions, respectively. The CTE of Sylgard matrix is taken as $3.1 \times 10^{-4} /{ }^{\circ} \mathrm{C}$ according to the previous work (Reinert and Luthy, 2005).

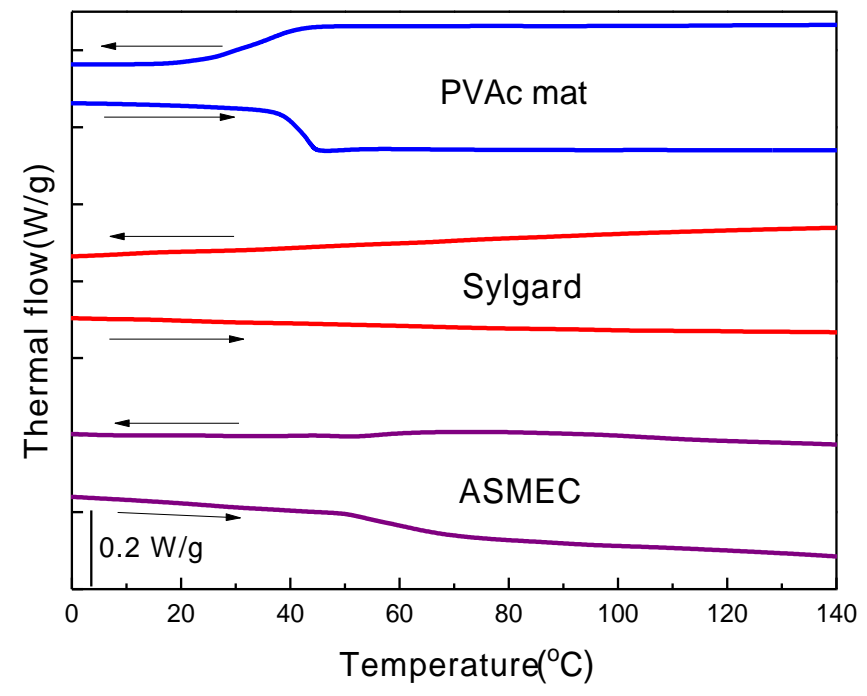

Figure 2. Differential Scanning Calorimetry (DSC) curves for a PVAc fibrous mat, neat Sylgard and single ASMEC lamina. The arrows in the figure indicate the increase/decrease of temperature. 


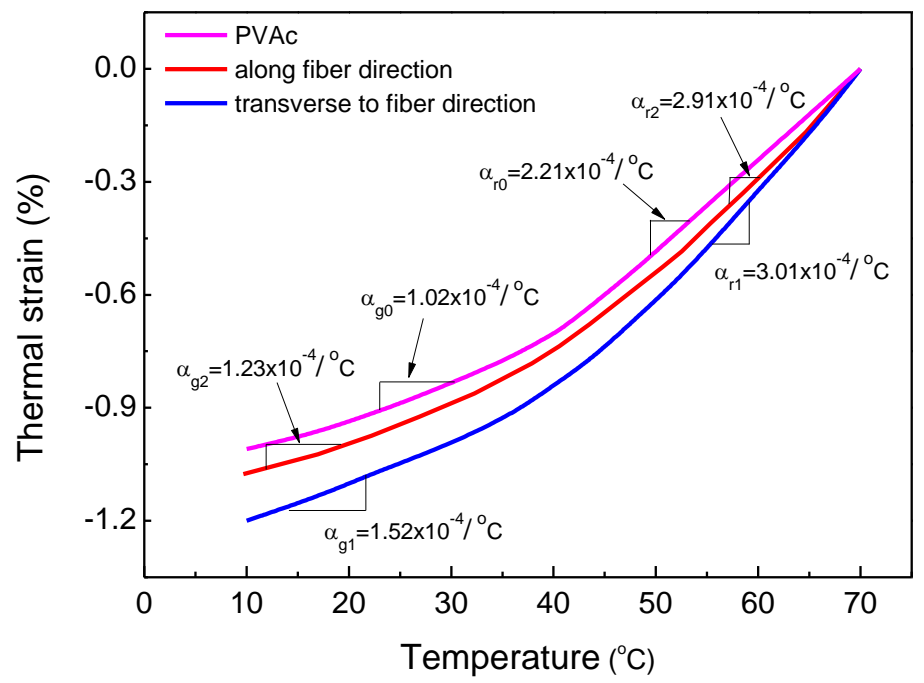

Figure 3. Thermal strain as a function of temperature for neat PVAc material and single layer ASMEC in two directions.

\subsection{Uniaxial tension of PAVc fibrous mat and single ASMEC lamina}

Mechanical tests on both the PVAc fibrous mat and the ASMEC were carried out using a Linkam TST 350 (Linkam Scientific Instruments Ltd., Tadworth, UK) tensile testing system. A custom made dog-bone die (ASTM D63 Type IV, scaled down by a factor of 4) was used to cut samples into dog-bone shapes with a gauge length of $6.25 \mathrm{~mm}$ and width of $1.5 \mathrm{~mm}$. Dog-bones were cut from the PVAc fibrous mats and single ASMEC lamina with $\theta=0^{\circ}, 22.5^{\circ}, 45^{\circ}$ and $90^{\circ}$, where $\theta$ is the angle between the direction parallel to the fibers and the loading direction. The samples were stretched at $25^{\circ} \mathrm{C}$ with a strain rate of $0.53 \% \mathrm{~s}^{-1}$ under the displacement control mode. For the single ASMEC lamina, in order to better observe deformation, larger samples (30mm (length) $\times 2.5 \mathrm{~mm}$ (width)) were cut. The samples were stretched at $1 \% \mathrm{~s}^{-1}$ at $25^{\circ} \mathrm{C}$ using the Linkam tensile testing system.

Fig.4a shows the engineering stress-engineering strain curves for PVAc fibrous mats. Table 1 lists the initial Young's moduli and the yield stresses of the fibrous mats with different fiber orientations. As expected, the general trend is that as fiber orientation angle increases, the fibrous mat becomes softer with lower yield stress. It is also noted that although the fibers are well 
aligned, the mat has load-carrying capability in the transverse direction. The reason for this might be due to the small amount of fibers that are out of the aligned direction, as evidenced in Fig. 1a, and some inter-fiber welding that inevitably occurs to some extent during electrospinning.

Table 1 Elastic modulus and yield stress of PVAc fibrous mat and single ASMEC lamina for different fiber orientations

\begin{tabular}{cccccccccccc}
\hline & \multicolumn{4}{c}{ PVAc fibrous mat } & \multicolumn{4}{c}{ single ASMEC lamina } \\
\cline { 2 - 10 } & $0^{\circ}$ & $22.5^{\circ}$ & $45^{\circ}$ & $67.5^{\circ}$ & $90^{\circ}$ & $0^{\circ}$ & $22.5^{\circ}$ & $45^{\circ}$ & $67.5^{\circ}$ & $90^{\circ}$ \\
\hline Elastic modulus (MPa) & 161.3 & 105.2 & 39.5 & 30.2 & 28.5 & 21.5 & 18.4 & 12.6 & 10.3 & 8.7 \\
Yield stress (MPa) & 10.5 & 5.4 & 2.4 & 2.1 & 1.9 & 3.3 & 1.9 & 1.4 & 0.8 & 0.5 \\
\hline
\end{tabular}

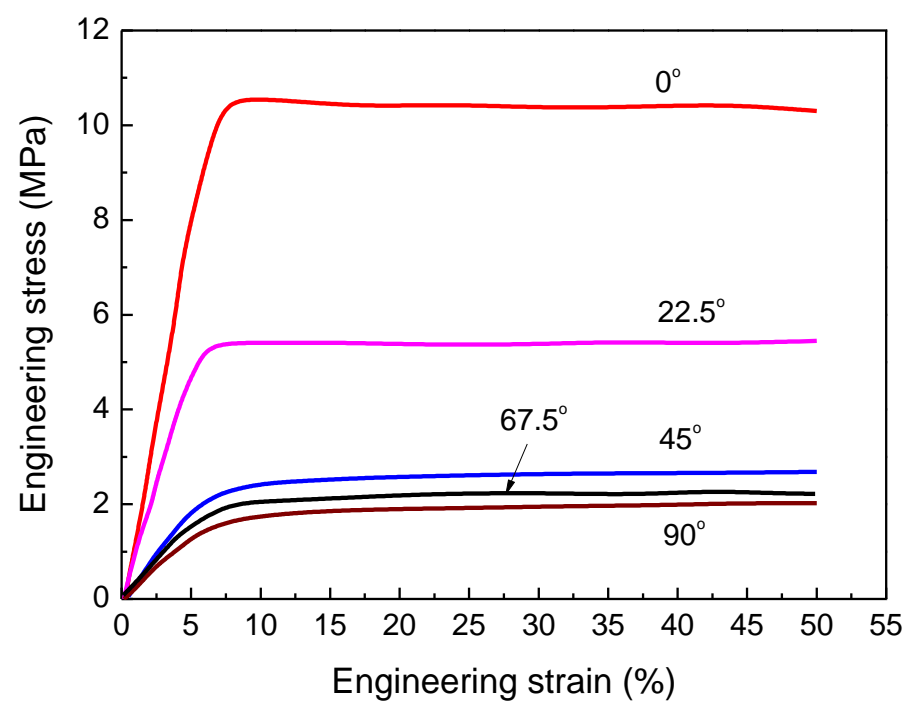

(a) 


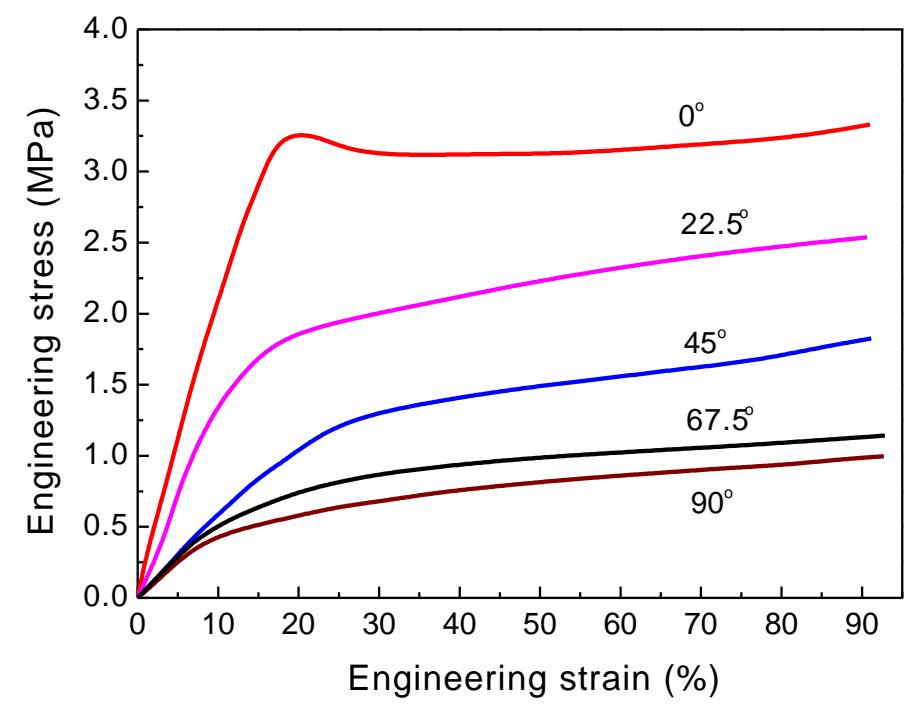

(b)

Figure 4. Measured engineering stress-engineering strain for (a) PVAc fibrous mat; (b) single ASMEC lamina with fiber orientation angles of $0^{\circ}, 22.5^{\circ}, 45^{\circ}, 67.5^{\circ}, 90^{\circ}$.

Fig.4b shows the engineering stress-engineering strain curves of the laminae, which exhibit a similar trend as that found in the fibrous mats. Table 1 shows the initial moduli and yield stresses for laminae of different fiber orientations. It can be found that the single ASMEC lamina has the Young's moduli in the range of $8.7-21.5 \mathrm{MPa}$ and the yield stresses of $0.52-3.3 \mathrm{MPa}$, which are lower than those for the PVAc fibrous mats. The reason for such a decrease in the moduli and the yield stresses will be discussed in the modeling section.

\subsection{Cold programmed anisotropic shape memory effects}

The cold programmed anisotropic shape memory effects were experimentally investigated. Here, the samples were cut into dog-bones with a gauge length of $6.25 \mathrm{~mm}$ and a width of 1.5 $\mathrm{mm}$. Prior to testing, the thermal histories of the samples were removed by heating to $60{ }^{\circ} \mathrm{C}$ for $20 \mathrm{~min}$, followed by cooling to room temperature $\left(25^{\circ} \mathrm{C}\right)$ for $5 \mathrm{~min}$. There were four steps in the thermomechanical cycle of the cold programmed shape memory experiments.

Step 1, the sample was stretched to a prescribed strain, $\quad{ }_{d}$, under force controlled loading 
at a low temperature, $T_{L}$.

Step 2, the sample was held isothermally for 1 min and then was unloaded.

Step 3, the temperature was heated from $T_{L}$ to $T_{H}$ at a heating rate of $\dot{T}$ for the shape recovery.

Step 4, the temperature was decreased to $T_{L}$ to complete the shape memory cycle.

Normally, $T_{\mathrm{L}}<T_{\mathrm{g}}<T_{\mathrm{H}}$. Figs. $5 \mathrm{a}$ and $5 \mathrm{~b}$ show the stress-strain and the strain-temperature behaviors, respectively, during the shape memory cycle with ${ }_{d}=75 \%, T_{L}=25^{\circ} \mathrm{C}, T_{H}=60^{\circ} \mathrm{C}$, and $\dot{T}=2^{\circ} \mathrm{C} / \mathrm{min}$. From Fig.5a, it can be seen that a large unrecovered strain is programmed upon unloading (Step 2). This strain can be maintained as long as the temperature is maintained at $T_{L}$. This unrecovered strain is used to define the shape fixity $R_{\mathrm{f}}$ as,

$$
R_{f}(\%)=\left({ }_{f} /{ }_{d}\right) 100,
$$

where $f$ is the strain upon unloading at room temperature. During heating, when the temperature is above the fiber glass transition temperature, the previously unrecovered strain can be recovered. The shape recovery ratio is defined as

$$
R_{r}(\%)=\left(\begin{array}{ll}
f & r
\end{array}\right) / f_{f} 100,
$$

where $\varepsilon_{r}$ is the strain after heating. Table 2 lists the shape fixity and shape recovery for ASMECs with different fiber orientation angles. It clearly shows that shape fixity strongly depends on the fiber orientation. In particular, fibers can provide the largest shape fixity when the loading direction is along the fiber direction. The fixity decreases as the fibers deviate from the loading direction but can still partially fix the shape even when they are perpendicular to the loading direction. This is similar to previous observations (Ge et al., 2013a) but is due to different reasons. In the previous case(Ge et al., 2013a), where the fiber orientation is precisely controlled by $3 \mathrm{D}$ printing, the shape fixity in the perpendicular direction is attributed to the Poisson's ratio effect. In the current case, as shown in Fig. 4a, the fiber mat can carry load and exhibits plastic 
deformation in the perpendicular direction. Therefore, the fixity in the perpendicular direction in the current study could be due to a combined effect of Poisson's ratio and non-perfectly aligned fibers.

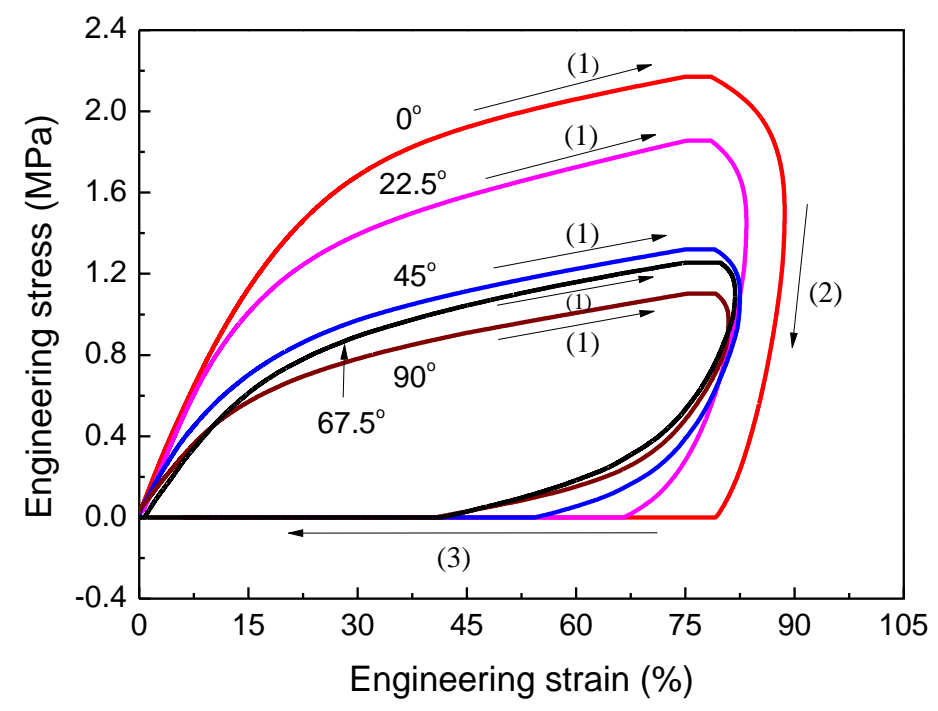

(a)

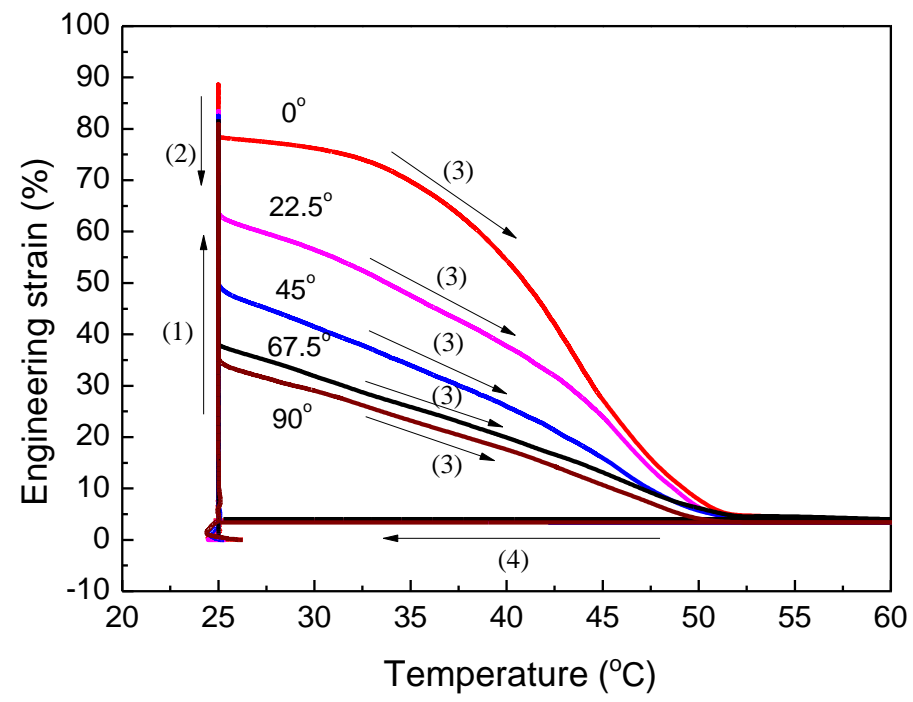

(b)

Figure 5. (a) Stress-strain curves and (b) Strain-temperature curves during a shape memory cycle for lamina with fiber orientation angles of $0^{\circ}, 22.5^{\circ}, 45^{\circ}, 67.5^{\circ}, 90^{\circ}$. A shape recovery cycle includes (1) loading at room temperature, (2) unloading at room temperature for fixing, (3) heating 
to $60{ }^{\circ} \mathrm{C}$ for recovery, and (4) cooling to room temperature.

Table 2. Measured shape fixity and recovery ratios for mechanically fixed shape-memory effects of ASMECs with different fiber angles.

\begin{tabular}{lccccc}
\hline & $0^{\circ}$ & $22.5^{\circ}$ & $45^{\circ}$ & $67.5^{\circ}$ & $90^{\circ}$ \\
\hline Fixity (\%) & 83.7 & 76.9 & 65.0 & 49.8 & 47.8 \\
Recovery ratio(\%) & 97.7 & 96.8 & 97.1 & 96.6 & 96.4 \\
\hline
\end{tabular}

\subsection{Uniaxial tension of ASMEC laminates}

The measured engineering stress-engineering strain curves for the laminated ASMEC system are plotted in Fig. 6. The laminated ASMECs show an obvious anisotropic stress-strain behavior. Due to the coupling between layers, the modulus and the yield stress for laminated ASMECs with angles $\bar{\theta}$ of $22.5^{\circ}, 45^{\circ}, 67.5^{\circ}$, and $90^{\circ}$ are larger than those of single layer ASMECs with fiber angles of $22.5^{\circ}, 45^{\circ}, 67.5^{\circ}$, and $90^{\circ}$. On the other hand, we find there is a decrease of the modulus and the yield stress for the laminate with angle of $0^{\circ}$. This could be because the adhesive layer (Sylgard) between the two laminae of ASMECs decreased the effective cross sectional area of the load-bearing portion (fiber-containing) of the laminate.

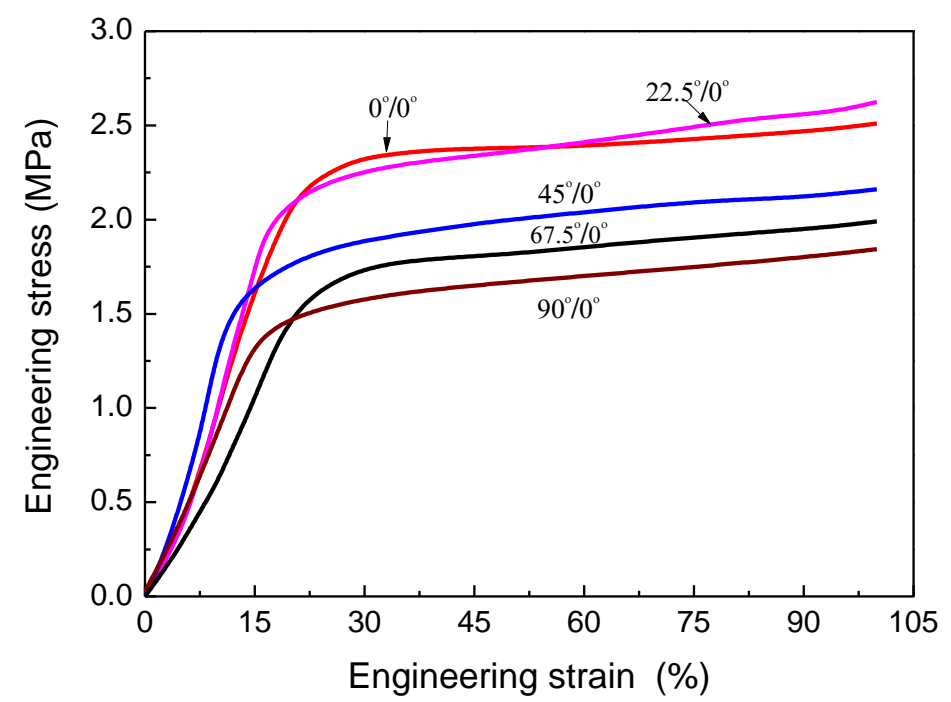

Figure 6. The measured engineering strain-engineering stress of bilayer composites under 
uniaxial stress-stretch. Fiber directions of top layers of composite are $0^{\circ}$ (red),22.5 (pink), $45^{\circ}$ (blue), $67.5^{\circ}$ (black), $90^{\circ}$ (purple), and the fiber directions of the bottom layers are $0^{\circ}$.
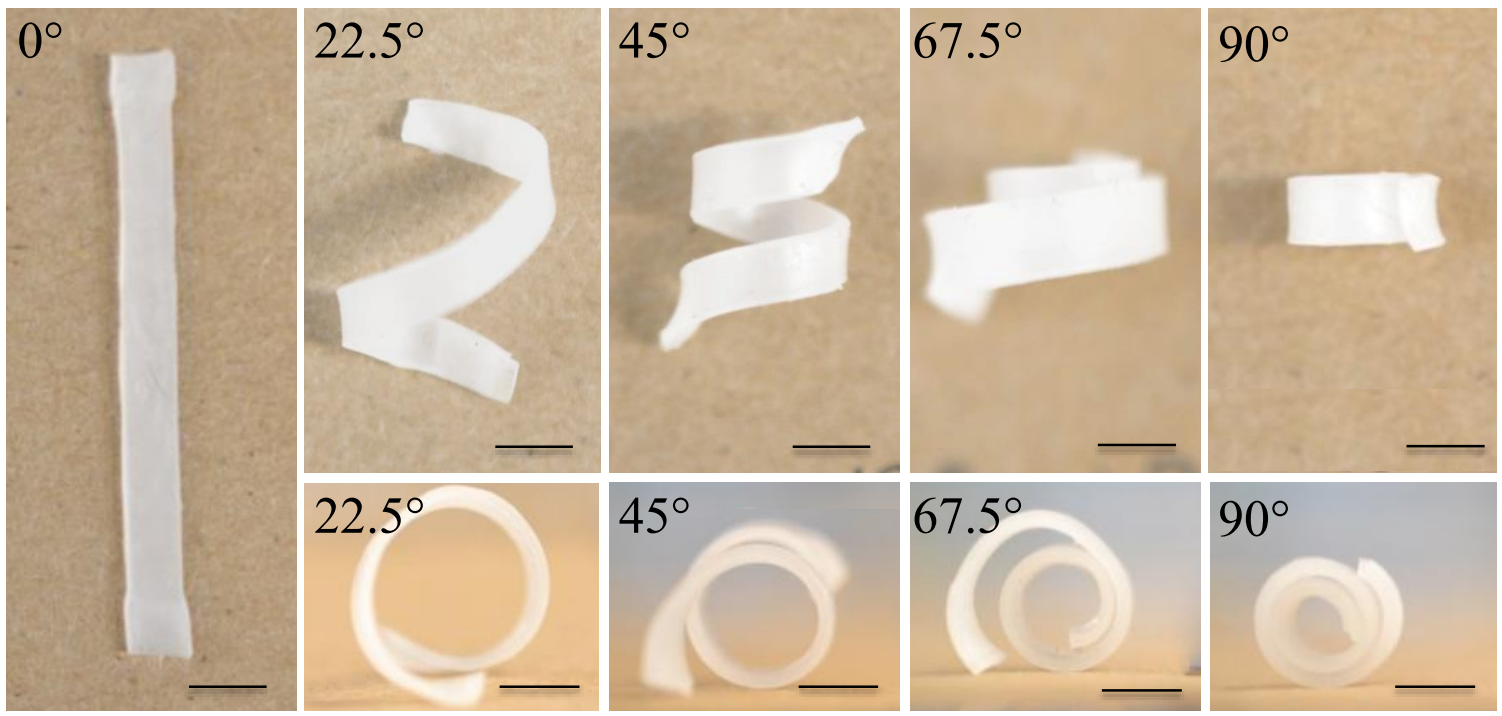

(a)

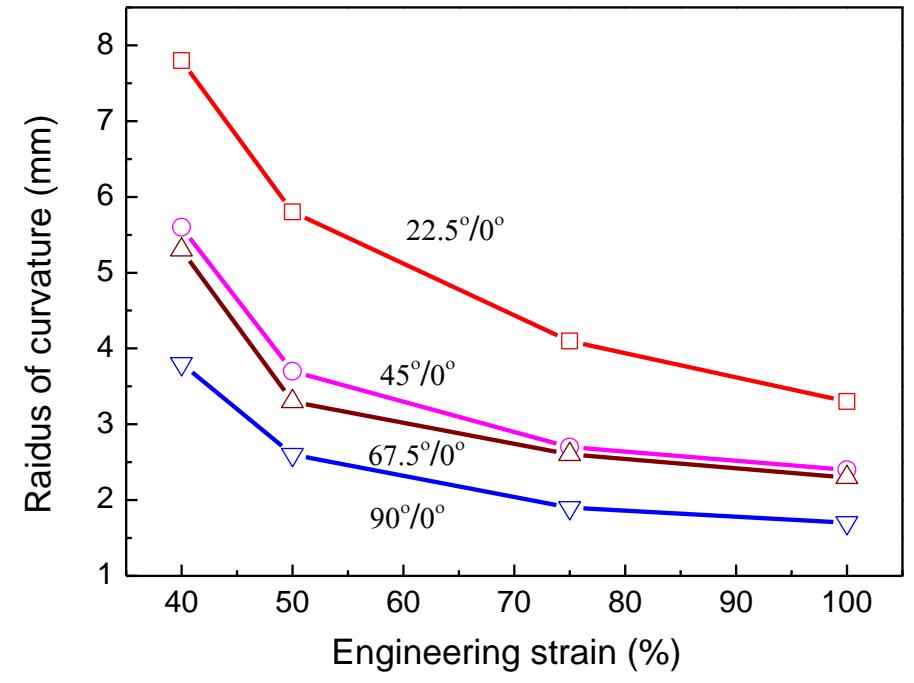

(b) 


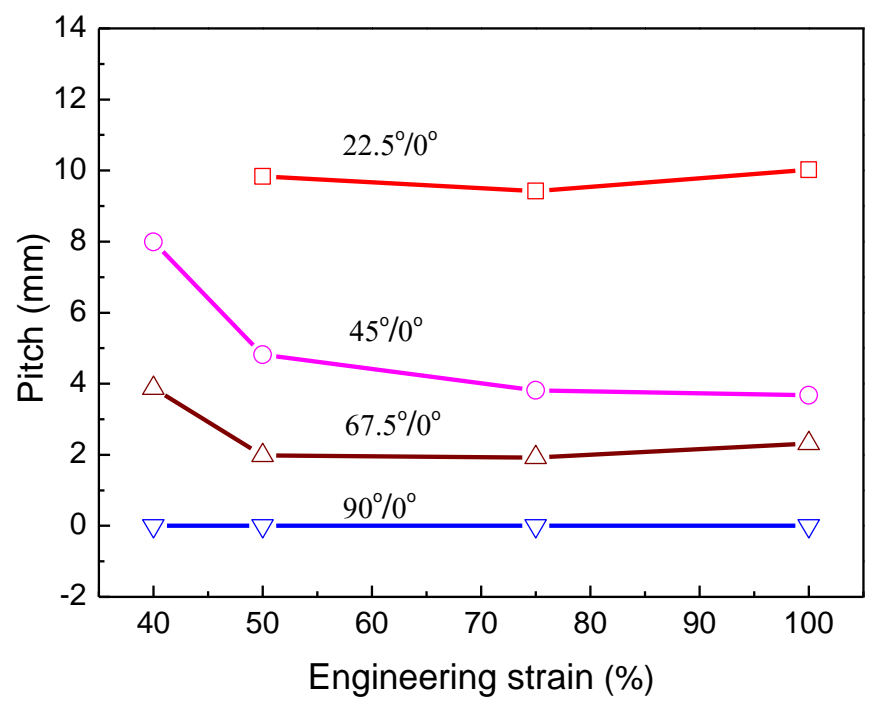

(c)

Figure 7. (a) Images of curled bilayer ASMEC strips stretched to 50\% strain. The pitch is in the corresponding top and the radius of curvature is in the bottom (scale bars are $4 \mathrm{~mm}$ )(Robertson et al., 2015). (b) The radius of curvature and (c) the pitch of ASMEC laminates as a function of stretched strain.

Upon unloading and releasing from the constraint of the grips, the strips generally distorted out of plane, twisting and bending to produce the complex helical shapes shown in the Fig.7a. The snapshots of the recovery are shown in Section 1 of the Supplementary Materials. It can be observed that as the orientation of the fibers in the top layer turns away from the bottom layer fiber direction (from parallel to perpendicular), the strip coils more tightly (toward the top) and the pitch decreases. In the case of $90^{\circ} / 0^{\circ}$, the strip becomes a coil whose interior is the $90^{\circ}$ lamina, with almost zero pitch. The observed bending and curling behaviors are due to the balance between the anisotropy in fixing ability for the PVAc fibers and the recovery force in the matrix. To quantify these changes, the radius of curvature and the pitch were measured and are presented in Fig. $7 \mathrm{~b}$ and c. The pitch was obtained by measuring the distance between consecutive peaks of the curled sample, and the radius of curvature was obtained by measuring the diameter of the sample in its curled state and dividing by 2 . It can be seen that the pitch and radius of curvature have strong dependence on the fiber orientation and the applied stretch and $\bar{\theta}$. It is also noted that no 
curvature measurement was observed for $\bar{\theta}=0^{\circ}$ since the samples remained in a flat state (as shown in the first figure of Fig.7a). Concerning the effect of strain, the sample curvatures increased (the radius of curvature decreased) monotonically with increasing strain (Fig. 7b), while the pitch was insensitive to strain (Fig. 7c).

\section{Model descriptions}

\subsection{Overall model for lamina}

In this section, we develop a constitutive model for the anisotropic time-temperature dependent behaviors for ASMECs. The thermal expansion is simply considered as an average of the two phases, the fiber and matrix. For the mechanical part, we explicitly split the contributions of the fiber mat and the matrix. A time-dependent thermomechanical constitutive model was developed previously for isotropic shape memory elastomeric composites (Ge et al., 2012), where the time dependence was due to the melt-crystal transition. The ASMEC studied in this paper is fundamentally different because its anisotropic time-dependent inelastic deformation comes from viscoplasticity. Moreover, an additional complication comes from the anisotropic deformation of the fibers.

Fig. 8 shows the 1-D analogy of the constitutive model. Anisotropic thermal expansion is considered. For the mechanical part, the matrix is considered to be a conventional elastomer represented by a neo-Hookean model (Nguyen et al., 2008b; Sun and Leong, 2004). Concerning the fibrous portion of the composites, tensile tests in the transverse direction showed load-bearing capacity despite microscopic observations of fibers being predominately aligned in one direction. The material stiffness in the transverse direction is mainly provided by the randomly oriented fibers (as shown in Fig.1a). Therefore, we further separate the fibrous mat into isotropic and anisotropic portions. The anisotropic portion characterizes the aligned fiber bundles with the anisotropic property and the isotropic part is considered for the average mechanical behavior of the randomly dispersed fibers. Further, we assume perfect bonding between the fibers and the matrix. Here, we note that PVAc is an amorphous uncrosslinked 
polymer; at temperatures above its $T_{\mathrm{g}}$, it becomes a melt that can flow(Ferry, 1980). Therefore, we use a Maxwell element to represents the time-dependent behavior of the PVAc. Here, it should also be noted that a single Maxwell element represents the viscoplastic behavior of the material, and cannot recover its original shape by itself, unless an external driving force is applied. At low temperature, the time constant for the dashpot is very large but can be reduced significantly by stress, thus enabling the plastic-like deformation behavior; at high temperature, the time constant becomes so low that the Maxwell element shows a liquid-like behavior.

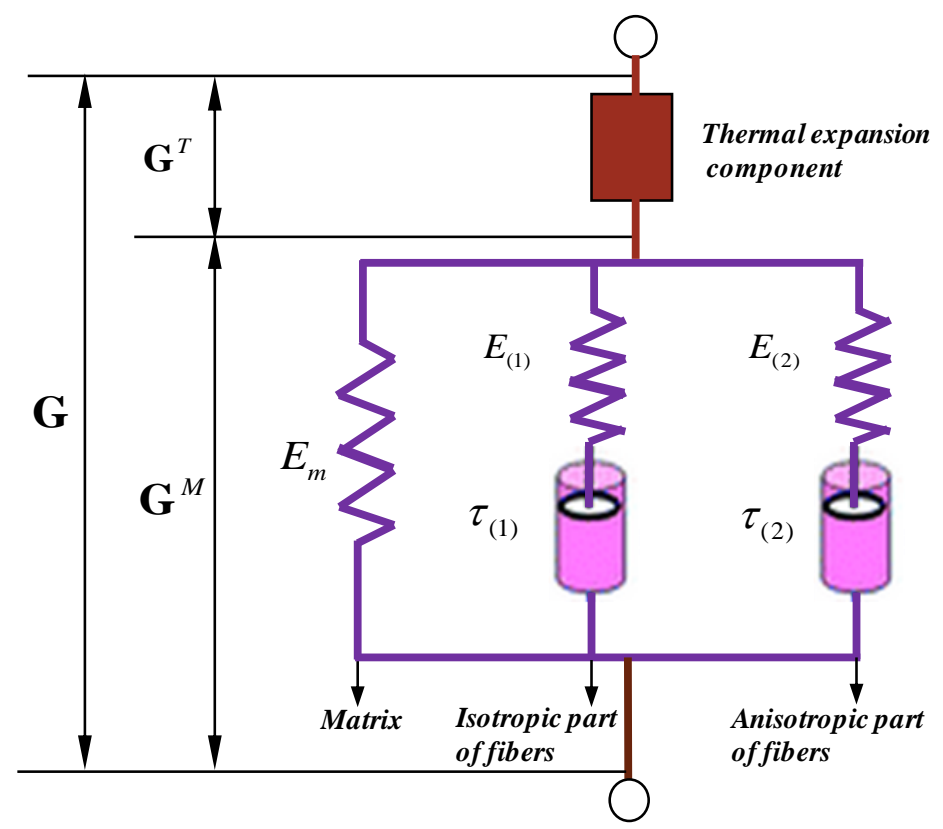

Figure 8. 1D analogy of the constitutive model for the ASMEC. The top block represents the thermal expansion/contraction. The left spring represents the matrix as an elastomer. The right two non-equilibrium branches represent fiber network, i.e., isotropic part and anisotropic part.

\subsection{Kinematics of the elastic-inelastic body}

The macroscopic deformation of the ASMEC is described by the deformation gradient tensor, $\mathbf{G}=\partial \mathbf{x} / \partial \mathbf{X}$, where $\mathbf{X}$ is the position vector of a material particle in the initial configuration, and $\mathbf{X}$ is the corresponding position vector of the particle in the current configuration. Following (Lion, 2000), we adopt the assumption that the thermomechanical deformation can be split locally into a stress-free thermal expansion and a mechanical 
stress-producing deformation as:

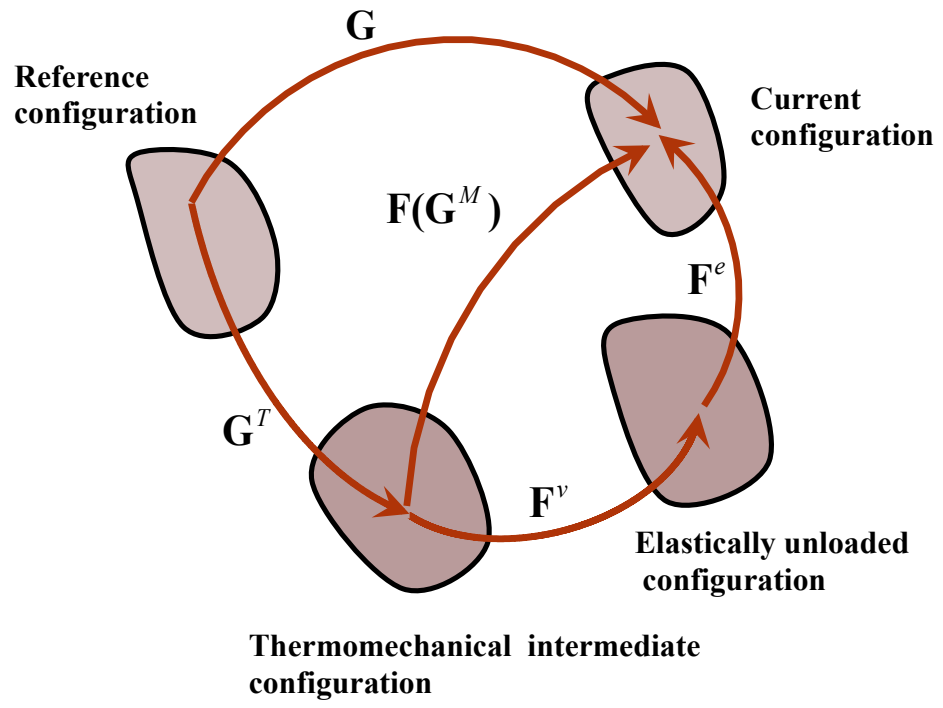

Figure 9. Thermomechanical decomposition of the deformation for ASMEC

$$
\mathbf{G}=\mathbf{G}^{M} \cdot \mathbf{G}^{T}
$$

where $\mathbf{G}^{M}$ is the mechanical deformation gradient; $\mathbf{G}^{T}$ is the deformation gradient related to stress-free thermal deformation as shown in Fig. 9.

For annotation convenience, we define $\mathbf{F} \quad \mathbf{G}^{M}$ as the mechanical deformation gradient in the remainder of this paper. The model for the viscoplastic deformation of the fiber mat starts with the multiplicative decomposition of the mechanical deformation gradient into elastic parts and inelastic parts for the isotropic and anisotropic part of the fiber mat as shown in Fig. 9,

$$
\mathbf{F}=\mathbf{F}_{(1)}^{e} \cdot \mathbf{F}_{(1)}^{v}=\mathbf{F}_{(2)}^{e} \cdot \mathbf{F}_{(2)}^{v}
$$

where subscripts 1 and 2 indicate the variables related to the isotropic and anisotropic parts of the fiber network (as shown in Fig. 8).

For convenience in further derivation, three unit structure tensors are defined for anisotropic fibers in the thermomechanical intermediate configuration, $\mathbf{M}_{0}$, the elastically unloaded configuration, $\tilde{\mathbf{M}}$, and the current configuration, $\overline{\mathbf{M}}$, as, 


$$
\mathbf{M}_{0}=\mathbf{a}_{0} \otimes \mathbf{a}_{0}, \quad \tilde{\mathbf{M}}=\tilde{\mathbf{a}} \otimes \tilde{\mathbf{a}}=\frac{\mathbf{F}_{(2)}^{v} \cdot \mathbf{M}_{0} \cdot \mathbf{F}_{(2)}^{v^{T}}}{\mathbf{C}_{(2)}^{v}: \mathbf{M}_{0}}, \quad \overline{\mathbf{M}}=\overline{\mathbf{a}} \otimes \overline{\mathbf{a}} .
$$

where $\mathbf{a}_{0}, \tilde{\mathbf{a}}$ and $\mathbf{a}$ are unit fiber directions defined in the above three configurations, respectively, and $\tilde{\mathbf{a}}=\lambda_{(2)}^{v^{-1}} \mathbf{F}_{(2)}^{v} \mathbf{a}_{0}, \overline{\mathbf{a}}=\lambda_{F}^{-1} \mathbf{F} \mathbf{a}_{0}$, where $\lambda_{F}=\left|\mathbf{F} \mathbf{a}_{0}\right|$ is the mechanical stretch of a fiber.

For later use, the following invariants associated with volume-preserving deformation are defined for matrix, isotropic and anisotropic part of fibers as,

$$
\begin{gathered}
\bar{I}_{1}=J^{-2 / 3} I_{1}=J^{-2 / 3} \operatorname{tr} \mathbf{C}, \bar{I}_{(1)_{1}}^{e}=\overline{\mathbf{C}}_{(i)}^{e}: \mathbf{I}=J_{(1)}^{e^{-2 / 3}} \mathbf{C}_{(1)}^{e}: \mathbf{I}, \\
\bar{I}_{(2)_{4}}^{e}=\overline{\mathbf{C}}_{(i)}^{e}: \tilde{\mathbf{M}}=J_{(2)}^{e^{-2 / 3}} \mathbf{C}_{(2)}^{e}: \tilde{\mathbf{M}}, \bar{I}_{(2)_{5}}^{e}=\overline{\mathbf{C}}_{(i)}^{e^{2}}: \tilde{\mathbf{M}}=J_{(2)}^{e^{-4 / 3}} \mathbf{C}_{(2)}^{e^{2}}: \tilde{\mathbf{M}} .
\end{gathered}
$$

where $\mathbf{C}, \mathbf{C}_{(1)}^{e}$ and $\mathbf{C}_{(2)}^{e}$ are the left Cauchy-Green tensor for the matrix, the isotropic and the anisotropic part of fiber, and $\mathbf{C}=\mathbf{F}^{T} \mathbf{F}, \mathbf{C}_{(i)}^{e}=J_{(i)}^{e^{-2 / 3}} \mathbf{F}_{(i)}^{e^{T}} \mathbf{F}_{(i)}^{e}(i=1,2)$. Here, all the variables are defined following a typical way in continuum mechanics, and more details on variable definitions are presented in Section 2 of Supplementary Material.

\subsection{The free energy}

A Helmholtz free-energy density function $=\left(\mathbf{C}, \mathbf{C}_{(1)}^{e}, \mathbf{C}_{(2)}^{e}, T\right)$ is defined for the thermomechanical state of the ASMEC, where $\mathbf{C}$ is the right Cauchy-Green mechanical deformation tensor associated with the matrix, $\mathbf{C}_{(1)}^{e}$ and $\mathbf{C}_{(2)}^{e}$ are the elastic deformation tensors of the isotropic and anisotropic parts of the fiber mat, respectively, and $T$ is temperature. For polymers, it is generally beneficial to decouple the total free-energy function $\Psi$ into a volumetric part $\left(\Psi_{V}\right)$ and an isochoric part $\left(\Psi_{I}\right)$ as

$$
\Psi\left(\mathbf{C}, \mathbf{C}_{(1)}^{e}, \mathbf{C}_{(2)}^{e}, T\right)=\Psi_{V}(J)+\Psi_{I}\left(\overline{\mathbf{C}}, \overline{\mathbf{C}}_{(1)}^{e}, \overline{\mathbf{C}}_{(2)}^{e}, T\right) .
$$

For the above energy function split, both the volumetric part and isochoric part can be further split into contributions from the Sylgard matrix and the fibrous mat weighted by their corresponding volume fractions. For the Sylgard matrix, it is reasonable to assume it is incompressible. We thus only consider volume variation attributed to the fiber mat in the current 
model. Therefore,

$$
\begin{gathered}
\Psi_{V}(J)=V_{F} \Psi_{V}^{F}(J), \\
\Psi_{I}=V_{M} \Psi_{I}^{M}(\overline{\mathbf{C}}, T)+V_{F}\left(\Psi_{I}^{F(\text { iso })}\left(\overline{\mathbf{C}}_{(1)}^{e}, T\right)+\Psi_{I}^{F(\text { aniso })}\left(\overline{\mathbf{C}}_{(2)}^{e}, T\right)\right),
\end{gathered}
$$

where $\Psi_{I}^{M}(\overline{\mathbf{C}}, T)$ is the isochoric contribution from the matrix, $\Psi_{I}^{F(i s o)}\left(\overline{\mathbf{C}}_{(1)}^{e}, T\right)$ and $\Psi_{I}^{F(\text { aniso) }}\left(\overline{\mathbf{C}}_{(2)}^{e}, T\right)$ are the isochoric contributions from the isotropic and anisotropic parts of the fibrous mat, and $V_{M}$ and $V_{F}$ are the volume fractions of the matrix and the fibers, respectively.

It is noted that a more general consideration of the energy split should include the interaction between fibers and matrix material in the fiber reinforced composites. For example, some recent works by Milani and Nemes (Milani and Nemes, 2004) and Guo and coworkers(Guo et al., 2006, 2007; Peng et al., 2006) showed that a better prediction of the material behavior could be obtained by including fiber-matrix shear interaction energy in the total free energy. However, in our work, we found that considering interaction effects does not offer a better prediction, but would make the model unnecessarily complicated, as more fitting parameters would have to be included. Therefore, the fiber-matrix interaction effect is neglected in the current model. The model and corresponding results with the consideration of fiber-matrix interaction are presented in Section 6 of the Supplementary Material. It is noted that although the volumetric part (Eq. (7a)) can contain elastic and inelastic deformation, the latter is less significant but is very difficult to be experimentally determined. We therefore only consider the elastic response of the volumetric part.

Under the thermodynamic framework, the total stress of the ASMEC, a combination of contributions from the matrix and fibers, can be defined as following. The detailed derivation procedure is presented in Section 3 of Supplementary Material.

$$
\mathbf{S}=p J \mathbf{C}^{-1}+\mathbf{S}_{M}+\mathbf{S}_{(1)}+\mathbf{S}_{(2)},
$$

where $\mathbf{S}_{M}, \mathbf{S}_{(1)}$ and $\mathbf{S}_{(2)}$ are the stresses for the matrix, the isotropic and the anisotropic part of the fiber mat, respectively. It should be noted that $\mathbf{S}$ is defined in the thermomechanical 
intermediate configuration. We call $\mathbf{S}$ the second Piola-Kirchhoff type stress in the rest of this paper, where the constitutive relations are developed in the thermomechanical intermediate configuration.

Following non-negative dissipation by the isotropic and anisotropic parts of the fibrous mat and by thermal flux, we have,

$$
\zeta_{(1)}=\frac{1}{2} \mathbf{M}_{(1)}: \mathbf{L}_{(1)}^{v} \geq 0, \quad \zeta_{(2)}=\frac{1}{2} \mathbf{M}_{(2)}: \mathbf{L}_{(2)}^{v} \geq 0, \quad \varsigma_{T}=-\frac{\mathbf{Q} \cdot \mathrm{Grad} T}{T \hbar} \geq 0
$$

where $\mathbf{M}_{(1)}$ and $\mathbf{M}_{(2)}$ are Mandel stress defined for the isotropic and anisotropic parts of fibrous mat. A detailed definition can be referred to Eq.(S22) in the Supplementary Material.

In Eq.(8), $p$ is the hydrostatic stress, and

$$
p=V_{F} \frac{\partial\left(\rho_{R T} \Psi_{V}\right)}{\partial J} .
$$

where, $V_{F}$ is the fiber volume fraction, which is introduced for the volume deformation of the fibrous mat.

The second Piola-Kirchhoff type stresses for different contributions from the matrix and the fiber mat in Eq.(8) are defined as

$$
\mathbf{S}_{M}=J^{-2 / 3} \tilde{\mathrm{P}}: \widehat{\mathbf{S}}_{M}, \quad \mathbf{S}_{(1)}=J_{(1)}^{e^{-2 / 3}} \tilde{\mathrm{P}}_{(1)}^{e}: \widehat{\mathbf{S}}_{(1)}, \quad \mathbf{S}_{(2)}=J_{(2)}^{e^{-2 / 3}} \tilde{\mathrm{P}}_{(2)}^{e}: \widehat{\mathbf{S}}_{(2)},
$$

where, $\quad \tilde{\mathbf{P}} \doteqdot \mathbf{I}-(1 / 3) \mathbf{C}^{-1} \otimes \mathbf{C}, \tilde{\mathbf{P}}_{(1)}^{e}=\mathbf{I}-(1 / 3) \mathbf{C}_{(1)}^{e^{-1}} \otimes \mathbf{C}_{(1)}^{e}, \tilde{\mathbf{P}}_{(2)}^{e}=\mathbf{I}-(1 / 3) \mathbf{C}_{(2)}^{e^{-1}} \otimes \mathbf{C}_{(2)}^{e} \cdot \widehat{\mathbf{S}}_{M} \quad$ is $\quad$ the $\quad$ stress contribution from the matrix, $\widehat{\mathbf{S}}_{(1)}$ and $\widehat{\mathbf{S}}_{(2)}$ are the stresses for the isotropic and anisotropic parts of fiber mat, and

$$
\begin{gathered}
\widehat{\mathbf{S}}_{M}=2 V_{M} \frac{\partial\left(\rho_{R T} \Psi_{I}^{M}\right)}{\partial \overline{\mathbf{C}}}, \\
\widehat{\mathbf{S}}_{(1)}=2 V_{F} \mathbf{F}_{(1)}^{v^{-1}} \cdot\left(\frac{\partial\left(\rho_{R T} \Psi_{I}^{F(\text { iso })}\right)}{\partial \overline{\mathbf{C}}_{(1)}^{e}}\right) \cdot \mathbf{F}_{(1)}^{v^{-T}}, \\
\widehat{\mathbf{S}}_{(2)}=2 V_{F} \mathbf{F}_{(2)}^{v^{-1}} \cdot\left(\frac{\partial\left(\rho_{R T} \Psi_{I}^{F(\text { aniso })}\right)}{\partial \overline{\mathbf{C}}_{(2)}^{e}}\right) \cdot \mathbf{F}_{(2)}^{v^{-T}} .
\end{gathered}
$$


We take the free energy for the volumetric deformation as

$$
\rho_{R T} \Psi_{V}(J)=\frac{1}{2} D(J-1)^{2}
$$

where $D$ is the bulk modulus.

By providing specific free energy functions for the matrix and the isotropic and anisotropic parts of the fibrous mat, the constitutive relations for ASMEC can be obtained. In the following, the specific free energy functions are given for the matrix and fibrous mat.

\subsection{Free energy function for matrix}

The elastomer matrix (Sylgard) is considered as an isotropic hyperelastic material, whose free energy is obtained using a neo-Hookean model

$$
\rho_{R T} \Psi_{I}^{M}\left(\bar{I}_{1}\right)=\frac{1}{2} \mu_{M}\left(\bar{I}_{1}-3\right) .
$$

$\mu_{m}$ is the shear modulus and is temperature dependent as $\mu_{m}=N k_{b} T$, where $N$ is the crosslinking density, $k_{b}$ is the Boltzmann constant and $T$ is temperature. From Eq. (14) and Eq. (12a), the stress in the matrix can be written as

$$
\widehat{\mathbf{S}}_{m}=\mu_{m} V_{M} \mathbf{I}
$$

\subsection{Free energy function for fibrous mat}

\subsubsection{Isotropic part}

For the non woven mat with randomly oriented fiber bundles, we choose the neo-Hookean model for the isotropic fraction of the fibrous mat as

$$
\rho_{R T} \Psi_{I}^{F(i s o)}\left(\bar{I}_{1}\right)=\frac{1}{2} \mu_{F(1)}\left(\bar{I}_{(1)_{1}}^{e}-3\right)
$$

where $\mu_{F(1)}$ is the shear modulus, and $\bar{I}_{(1)_{1}}^{e}$ is the first invariant defined in Eq. (10a).

\subsubsection{Anisotropic part}

To capture the anisotropic part, we follow the approach by Kao(2011). For anisotropic materials, the fiber stretch in the fiber direction, $\mathbf{a}$, is 


$$
\lambda_{f}^{e}=\sqrt{\frac{\bar{I}_{(2)_{4}}^{e}}{3}} .
$$

However, it was found that the above definition of chain stretch represents a strong interaction between the direction $\mathbf{a}_{0}$ and its lateral direction. On the basis of observations in Fig. 1a, it is reasonable to assume that the interactions between the fiber direction and the lateral direction are relatively weak. To capture the effect of weak interaction, we therefore define an invariant(Kao, 2011),

$$
\bar{I}_{a 2}^{e}=\frac{1}{2}\left(\bar{I}_{(2)_{4}}^{e^{2}}-\bar{I}_{(2)_{5}}^{e^{2}}\right),
$$

which resembles the second invariant in the isotropic material analogy. By introducing an index, $c$, the following new stretch measurement is defined as

$$
\tilde{\lambda}_{f}^{e}=\sqrt[4]{\left(\bar{I}_{(2)_{4}}^{e}\right)^{2}+c \bar{I}_{a 2}^{e}} .
$$

here, the index $c$ is used to adjust interactions of the fiber and its lateral direction. We define the following strain energy for the anisotropic part of the fibrous mat as

$$
\rho_{R T} \Psi_{I}^{F(\text { aniso })}\left(\bar{I}_{(2)_{4}}^{e}, \bar{I}_{(2)_{5}}^{e}\right)=\frac{1}{2} \mu_{F(2)}\left(\left(\tilde{\lambda}_{f}^{e}\right)^{2}-1\right)^{2}
$$

By using the relations Eq. (12b,c) and free energy formations of Eqs. (16) and (20), we can write the stresses for the isotropic and anisotropic parts of the fibrous mat as

$$
\begin{gathered}
\widehat{\mathbf{S}}_{(1)}=\tilde{\mu}_{F(1)}\left(\mathbf{F}_{(1)}^{v^{-1}} \cdot \mathbf{F}_{(1)}^{v^{-T}}\right), \\
\widehat{\mathbf{S}}_{(2)}=\mathbf{F}_{(2)}^{v^{-1}} \cdot\left(\tilde{\mu}_{F(2)} \tilde{\mathbf{M}}+\tilde{\mu}_{F(3)} \overline{\mathbf{C}}_{(2)}^{e}: \tilde{\mathbf{M}}\right) \cdot \mathbf{F}_{(2)}^{v^{-T}},
\end{gathered}
$$

where the reduced material coefficients are

$$
\begin{gathered}
\tilde{\mu}_{F(1)}=V_{F} \mu_{F(1)}, \\
\tilde{\mu}_{F(2)}=2 V_{F} \mu_{F(2)}\left(\tilde{\lambda}_{f}^{e^{2}}-1\right) \tilde{\lambda}_{f}^{e} \frac{\partial \tilde{\lambda}_{f}^{e}}{\partial \overline{\boldsymbol{I}}_{(2)_{4}}^{e}}, \\
\tilde{\mu}_{F(3)}=2 V_{F} \mu_{F(2)}\left(\tilde{\lambda}_{f}^{e 2}-1\right) \tilde{\lambda}_{f}^{e} \frac{\partial \tilde{\lambda}_{f}^{e}}{\partial \overline{\boldsymbol{I}}_{(2)_{5}}^{e}},
\end{gathered}
$$


where $\mu_{F(1)}$ and $\mu_{F(2)}$ are the shear moduli of the isotropic part and anisotropic parts, respectively, as shown in Eq. (16) and Eq. (20), which can be fitted from experiment results.

As shown in Table 1, the ASMEC has a lower modulus than the fibrous mat. That is because the infilled Sylgard dilutes (expands) the fiber network, lowering fiber volume fraction, and consequently decrease the modulus. Therefore, we introduce an index to depict the decrease in modulus of the fibrous mat, i.e., $\mu_{F(1)}^{\prime}=\omega \mu_{F(1)}, \mu_{F(2)}^{\prime}=\omega \mu_{F(2)}$. Here, $\mu_{F(1)}$ and $\mu_{F(2)}$ are the moduli fitted from experiments on the isotropic and anisotropic parts of the fibrous mat, and $\mu_{F(1)}^{\prime}$ and $\mu_{F(2)}^{\prime}$ are the reduced moduli; $\omega$ is a parameter representing the Sylgard infiltration induced reduction of modulus. The reduced modulus of the fibrous mat in the single ASMEC lamina are modified as

$$
\begin{gathered}
\tilde{\mu}_{F(1)}=V_{F} \mu_{F(1)}^{\prime}, \\
\tilde{\mu}_{F(2)}=2 V_{F} \mu_{F(2)}^{\prime}\left(\tilde{\lambda}_{f}^{e^{2}}-1\right) \tilde{\lambda}_{f}^{e} \frac{\partial \tilde{\lambda}_{f}^{e}}{\partial \overline{\boldsymbol{I}}_{(2)_{4}}^{e}}, \\
\tilde{\mu}_{F(3)}=2 V_{F} \mu_{F(2)}^{\prime}\left(\tilde{\lambda}_{f}^{e 2}-1\right) \tilde{\lambda}_{f}^{e} \frac{\partial \tilde{\lambda}_{f}^{e}}{\partial \overline{\boldsymbol{I}}_{(2)_{5}}^{e}} .
\end{gathered}
$$

Then, the total stress of Eq. (8) from matrix and fiber can be written as

$$
\mathbf{S}=p J \mathbf{C}^{-1}+\underbrace{J^{-2 / 3} \tilde{\mathbf{P}}: \widehat{\mathbf{S}}_{M}}_{\mathbf{S}_{M}}+\underbrace{J_{(1)}^{e^{-2 / 3}} \tilde{\mathrm{P}}_{(1)}^{e}: \widehat{\mathbf{S}}_{(1)}}_{\mathbf{S}_{(1)}}+\underbrace{J_{(2)}^{e^{-2 / 3}} \tilde{\mathrm{P}}_{(2)}^{e}: \widehat{\mathbf{S}}_{(2)}}_{\mathbf{S}_{(2)}},
$$

where, $\quad p$ is hydrostatic stress and $p=2 V_{F} D(J-1), \mathbf{S}_{M}, \mathbf{S}_{(1)}$ and $\mathbf{S}_{(2)}$ are the second Piola-Kirchhoff type stress for the matrix and the isotropic and anisotropic parts of the fiber mat. $J_{(1)}^{e}$ and $J_{(2)}^{e}$ are the volume deformations of isotropic and anisotropic parts of fiber mat, and relation $J_{(1)}^{e}=J_{(2)}^{e}=J$ is considered.

\subsection{Evolution equations}

Kinetic laws are required to describe the evolution of the material system, namely the heat 
flux and the viscous deformation of the fibers. Further, the laws must satisfy the constraints in Eq. (9). In current analysis, we assume sufficiently slow change of temperature in the heating and cooling processes, and thus the dissipation by the thermal flux is neglected. We separately present the evolution equations for viscous deformation of isotropic portion and anisotropic portion of fibers in the following.

\subsubsection{Viscous deformation for isotropic portion of fibrous mat}

As for the kinetics of the viscous deformation for the isotropic part, the first inequality of Eq. (9) can be rewritten in the current configuration as(Nedjar, 2007; Nguyen et al., 2007; Reese and Govindjee, 1998)

$$
-\tau_{(1)}: \frac{1}{2}\left(L_{v} \mathbf{b}_{(1)}^{e}\right) \mathbf{b}_{(1)}^{e^{-1}} \geq 0
$$

where the notation $L_{v} \mathbf{b}_{(1)}^{e}$ stands for the Lie derivative of the tensor $\mathbf{b}_{(1)}^{e}$, and the Kirchhoff stress and left Green strain are defined as

$$
\tau_{(1)}=2 \mathbf{F}_{(1)}^{e}\left(\frac{V_{F} \partial\left(\rho_{R \theta} \partial \Psi_{I}^{F(i s o)}\right)}{\partial \overline{\mathbf{C}}_{(1)}^{e}}: \frac{\partial \overline{\mathbf{C}}_{(1)}^{e}}{\partial \mathbf{C}_{(1)}^{e}}\right) \mathbf{F}_{(1)}^{e^{T}}, \quad \mathbf{b}_{(1)}^{e}=\mathbf{F}_{(1)}^{e} \cdot \mathbf{F}_{(1)}^{e^{T}}
$$

A sufficient condition fulfilling the first inequality of Eq. (9) is to make it a positive definite quadratic form. This choice gives the evolution equation(Reese and Govindjee, 1998),

$$
-\frac{1}{2}\left(L_{v} \mathbf{b}_{(1)}^{e}\right)=\frac{\tau_{(1)} \cdot \mathbf{b}_{(1)}^{e}}{2 \mu_{(1)} \tau_{(1)}} .
$$

\subsubsection{Viscous deformation for anisotropic portion of fibrous mat}

The second inequality of Eq. (9) pertaining to the anisotropic part of the fiber can be rewritten as (Nedjar, 2007)

$$
\varsigma_{(2)}=\mathbf{M}_{(2)}: \tilde{\mathbf{M}} \frac{\dot{\lambda}_{(2)}^{v}}{\lambda_{(2)}^{v}} \geq 0
$$

Here, the relations $\mathbf{L}_{(2)}^{v}=\dot{\mathbf{F}}_{(2)}^{v} \mathbf{F}_{(2)}^{v^{-1}}$, and $\mathbf{F}_{(2)}^{v}=\lambda_{(2)}^{v} \tilde{\mathbf{a}} \otimes \mathbf{a}_{0}$ are applied.

Following the assumption in (Nedjar, 2007), there exists a viscoplastic potential, $\phi_{v i s}\left(\mathbf{M}_{(2)}\right)$, 
expressed in the thermomechanical intermediate configuration, such that

$$
\tilde{\mathbf{M}} \frac{\dot{\lambda}_{(2)}^{v}}{\lambda_{(2)}^{v}}=\frac{\partial \phi_{v i s}\left(\mathbf{M}_{(2)}\right)}{\partial \mathbf{M}_{(2)}} \Rightarrow \dot{\lambda}_{(2)}^{v} \tilde{\mathbf{M}}=\lambda_{(2)}^{v} \frac{\partial \phi_{v i s}\left(\mathbf{M}_{(2)}\right)}{\partial \mathbf{M}_{(2)}}
$$

For the current problem, this viscoplastic potential is chosen as a simple quadratic expression (Nedjar, 2007),

$$
\phi_{v i s}\left(\mathbf{M}_{(2)}\right)=\frac{\left(\tilde{\mathbf{M}} \cdot \mathbf{M}_{(2)} \cdot \tilde{\mathbf{M}}\right):\left(\tilde{\mathbf{M}} \cdot \mathbf{M}_{(2)} \cdot \tilde{\mathbf{M}}\right)}{2 \mu_{(2)} \tau_{(2)}} .
$$

Then, Eq. (28) reduces to the following single scalar-valued evolution equation of the internal variable $\lambda_{f a}^{v}$,

$$
\dot{\lambda}_{(2)}^{v}=\frac{\lambda_{(2)}^{v}}{\mu_{(2)} \tau_{(2)}}\left(\mathbf{M}_{(2)}: \tilde{\mathbf{M}}\right)
$$

where the relation $\tilde{\mathbf{M}}^{n}=\tilde{\mathbf{M}}$ is applied for any value of the power $n$ since $\tilde{\mathbf{M}}$ is idempotent (Nedjar, 2007).

\subsection{Relaxation time and time-temperature shift factor}

The nonlinear viscoelastic and viscoplastic behaviors of amorphous polymers were studied intensively in the past. Broadly speaking, there are essentially two modeling approaches. One large group of models(Arruda and Boyce, 1993; Bergstrom and Boyce, 1998; Dupaix and Boyce, 2007; Nguyen et al., 2008a; Qi and Boyce, 2005; Qi et al., 2008; Srivastava et al., 2010a; Srivastava et al., 2010b) addresses viscous (or plastic) flow directly and is based on the work by Boyce et al(Boyce et al., 1988), who considers that the plastic flow in the material is due to the activation of kinks in the polymer structures. The Boyce model was later proved to be able of capturing viscoelastic behaviors as well(Reese and Govindjee, 1998). Another group of models(Adolf et al., 2004; Arzoumanidis and Liechti, 2003; Knauss and Emri, 1987; Knauss and Emri, 1981; Popelar and Liechti, 2003) considers the nonlinear response of polymers to be due to nonlinear viscoelasticity where the relaxation time is a function of not only temperature, but also dilatation, shape distortion, and shear stress. From this modeling concept, the so-called "plastic" 
deformation in amorphous polymers at low temperatures is recoverable when the temperature is raised above the glass transition temperature. This is because the large deformation at low temperature causes the material's relaxation time to be temporarily decreased to relax the stress (or excessive energy). Once the stress is released, the relaxation time increases again, and thus, the material exhibits "plastic" deformation. Raising the temperature reduces the relaxation time; if the polymer has a crosslinking network, this leads to the recovery of the polymer. Therefore, in this modeling approach, viscoplastic deformation is an exhibition of thermoviscoelastic behaviors of polymers under some special thermo-mechanical loading conditions. Following this concept, we defined the relaxation time for the nonequilibrium parts as (Westbrook et al., 2011)

$$
\tau_{(i)}=\tau_{0(i)} \alpha(T) \exp \left(-\frac{\Delta G}{k_{B} T} \frac{\sigma_{e(i)}}{s_{(i)}}\right) \quad(i=1,2),
$$

where the subscript $(i=1,2)$ indicates the variables associated with the isotropic and anisotropic parts of the fiber mat, respectively; $\alpha(T)$ is the time-temperature superposition (TTS) shift factor and $\tau_{0(i)}$ are the relaxation times at the reference temperature when $\alpha(T)=1 ; \Delta G$ is the activation energy and $s_{(i)}$ are the athermal shear strengths representing the resistance to the viscoplastic shear deformation in the material; $\sigma_{e(i)}$ are the equivalent stresses for the viscous evolution, defined as $\sigma_{e(i)}=\left[\left(\mathbf{M}_{(i)}^{\prime}: \mathbf{M}_{(i)}^{\prime}\right) / 2\right]^{1 / 2}$ (Westbrook et al., 2011), where $\mathbf{M}_{(i)}^{\prime}=\mathbf{M}_{(i)}-1 / 3 \operatorname{tr}\left(\mathbf{M}_{(i)}\right) \mathbf{I}$ and $\mathbf{M}_{(i)}$ are the Mandel stresses. Eq. (32) combines the approaches of Knauss and Emri(Knauss and Emri, 1987; Knauss and Emri, 1981) and Boyce et al(Boyce et al., 1988) by considering the shifting due to shear stress as the activation of kinks in the amorphous polymer structures.

In order to adequately account for the softening effects, the evolution rule for $s_{(i)}$ is defined as

$$
\dot{s}_{(i)}=h_{0}\left(1-s_{(i)} / s_{s(i)}\right) \dot{\gamma}_{(i)}^{v}, \quad s_{(i)}=s_{0(i)} \quad \text { when } \gamma_{(i)}^{v}=0
$$


where $s_{0(i)}$ are the initial values of the athermal shear strength, $s_{s(i)}$ are the saturation values, $h_{0}$ is the slope of the yield variation with respect to plastic strain, $\dot{\gamma}_{(i)}^{v}=\sigma_{e(i)} /\left(G_{(i)} \tau_{(i)}\right)$.

Following O'Connell and McKenna(O'Connell and McKenna, 1999), the influence of temperature on the viscoelastic behavior depends on whether the material temperature is above or below a temperature, $T_{s}$. At temperatures above $T_{s}$, the Williams-Landel-Ferry(WLF) equation(Williams et al., 1955) is used

$$
\log \alpha(T)=-\frac{C_{1}\left(T-T_{M}\right)}{C_{2}+\left(T-T_{M}\right)},
$$

where $C_{1}$ and $C_{2}$ are material constants and $T_{M}$ is the WLF reference temperature. As for temperature below $T_{s}$, the temperature shift factor, $\alpha(T)$, is set to follow Arrhenius-type behavior (DiMarzio and Yang, 1997), with

$$
\ln \alpha(T)=-\frac{A F_{c}}{k_{B}}\left(\frac{1}{T}-\frac{1}{T_{g}}\right),
$$

where $A$ and $F_{c}$ are material constants and $k_{B}$ is Boltzman's constant. Here, $T_{s}$ is calculated by equating $\alpha(T)$ in Eqs. (34) and (35).

\subsection{Thermal deformation}

From the thermal expansion experiment on the single layer ASMEC in section 2.2, the fiber reinforced ASMEC shows anisotropic thermal expansion behavior. In this section, we calculate the anisotropic coefficients of thermal expansion of the ASMEC by considering isotropic thermal deformation of the PVAc material and Sylgard matrix, respectively.

\subsubsection{CTE of PVAc}

When temperature changes, an isotropic, but nonlinear, thermal expansion behavior of the PVAc material is considered according to Lion(Lion, 2000). The thermal expansion is chosen as (Westbrook et al., 2011), 


$$
J_{T}=\frac{V(T, t)}{V_{0}}
$$

where $V(T, t)$ is the volume at time $t$ and temperature $T \cdot V_{0}$ is the reference volume at the reference temperature $T_{0}$. Defining the degree of the volume departure from equilibrium, $\delta$, as

$$
\delta=\frac{V(T, t)}{V_{e q}(T)}-1
$$

where $V_{e q}(T)$ is equilibrium volume, which is considered to be the material volume if the experimental timescale was infinitely long. It is considered as a linear extrapolation of the temperature dependence of the equilibrium volume above $T_{g}$ and is set as (Tool, 1946, 1948)

$$
V_{e q}(T)=\left[1+3 \alpha_{r}\left(T-T_{0}\right)\right] V_{0}
$$

where $\alpha_{r}$ is the linear CTE in the rubbery state. The total volume change is given by

$$
J_{T}=\left[1+3 \alpha_{r}\left(T-T_{0}\right)\right](1+\delta)
$$

where $\delta$ is calculated by the well-known KAHR 33-parameter(Kovacs et al., 1979, 1996).

From Eq. (39), we define the equivalent CTE for nonlinear thermal expansion of PVAc fibers as

$$
\bar{\alpha}_{f}=\frac{\left[1+3 \alpha_{r}\left(T-T_{0}\right)\right](1+\delta)}{\left(T-T_{0}\right)} .
$$

\subsubsection{Anisotropic CTE of ASMEC}

Many analytical models exist for the prediction of the bounds for effective coefficients of thermal expansion for unidirectional fiber reinforced composites with isotropic or anisotropic phases (Kardeniz and Kumlutas, 2007; Rosen and Hashin, 1970; Schapery, 1968). Following work in (Schapery, 1968), the thermal expansion coefficient of the anisotropic ASMEC along the fiber direction is defined as

$$
\alpha_{1}=\frac{E_{f(\text { aniso })} \bar{\alpha}_{f} v_{f}+\left(E_{m} \alpha_{m} v_{m}+E_{f(i s o)} \bar{\alpha}_{f} v_{f}\right)}{E_{f(\text { aniso })} v_{f}+\left(E_{m} v_{m}+E_{f(i s o)} v_{f}\right)}
$$


and the thermal expansion coefficient transverse to fiber direction is defined as

$$
\begin{aligned}
\alpha_{2}= & \left(1+V_{f(\text { aniso })}\right) \bar{\alpha}_{f} v_{f}+\left(1+V_{m}\right) \alpha_{m} v_{m}+\left(1+V_{f(\text { iso })}\right) \bar{\alpha}_{f} v_{f} \\
& -\alpha_{1}\left(V_{f(\text { aniso })} v_{f}+V_{m} v_{m}+V_{f(\text { iso })} v_{f}\right)
\end{aligned}
$$

where $\bar{\alpha}_{f}$ and $\alpha_{m}$ are the reduced equivalent thermal expansion coefficients of PVAc (Eq.40)

and the Sylgard matrix; $E_{f(i s o)}, E_{f(a n i s o)}$ and $E_{m}$ are the moduli of the isotropic and anisotropic parts of the fibrous mat and of the matrix, respectively; $v_{f}$ and $v_{m}$ are the Poisson's ratios of the PVAc fibers and the Sylgard matrix; $V_{f(\text { iso })}, V_{f(\text { aniso })}$ and $v_{m}$ are the volume fractions of the isotropic and anisotropic parts of the fibrous mat and of the Sylgard matrix, respectively.

\section{Model results and discussions}

A summary of current model is listed in Table 3, and the constitutive relations were implemented into a MATLAB program. In this section, we first show the process of material parameter identification. The parameters are then used to predict the shape memory behavior, which is compared with the experimental results to validate the model. Finally, we study the non-affine shape change from cold programming. We also conduct parametric studies to explore how the non-affine deformation can be influenced by different design parameters.

Table 3 Summary of the model

Total mechanical stress

$$
\mathbf{S}=p J \mathbf{C}^{-1}+\underbrace{J^{-2 / 3} \tilde{\mathrm{P}}_{M}: \widehat{\mathbf{S}}_{M}}_{\mathbf{S}_{M}}+\underbrace{J_{(1)}^{e^{-2 / 3}} \tilde{\mathrm{P}}_{(1)}^{e}}_{\mathbf{S}_{(1)}}: \widehat{\mathbf{S}}_{(1)}+\underbrace{J_{(2)}^{e^{-2 / 3}} \tilde{\mathrm{P}}_{(2)}^{e}}_{\mathbf{S}_{(2)}}: \widehat{\mathbf{S}}_{(2)}
$$

Matrix Isotropic part of fiber $\quad$ Anisotropic part of fiber

$$
\widehat{\mathbf{S}}_{m}=\mu_{m} V_{M} \mathbf{I} \quad \widehat{\mathbf{S}}_{(1)}=\tilde{\mu}_{F(1)}\left(\mathbf{F}_{(1)}^{v^{-1}} \cdot \mathbf{F}_{(1)}^{v^{-T}}\right) \quad \widehat{\mathbf{S}}_{(2)}=\mathbf{F}_{(2)}^{v^{-1}} \cdot\left(\tilde{\mu}_{F(2)} \tilde{\mathbf{M}}^{-\tilde{\mu}_{F(3)}} \overline{\mathbf{C}}_{(2)}^{e}: \tilde{\mathbf{M}}\right) \cdot \mathbf{F}_{(2)}^{v^{-T}}
$$

Deformation gradient

$$
\mathbf{F}=\mathbf{F}_{(1)}^{e} \cdot \mathbf{F}_{(1)}^{v}, \mathbf{b}_{(1)}^{e}=\mathbf{F}_{(1)}^{e} \cdot \mathbf{F}_{(1)}^{e^{\tau}} \quad \mathbf{F}=\mathbf{F}_{(2)}^{e} \cdot \mathbf{F}_{(2)}^{v}, \lambda_{(2)}^{v} \tilde{\mathbf{a}}=\mathbf{F}_{(2)}^{v} \mathbf{a}_{0}
$$

Viscoplastic

deformation

$$
-\frac{1}{2}\left(L_{v} \mathbf{b}_{(1)}^{e}\right)=\frac{\boldsymbol{\tau}_{(1)} \cdot \mathbf{b}_{(1)}^{e}}{2 \mu_{(1)} \tau_{(1)}} \quad \quad \dot{\lambda}_{(2)}^{v}=\frac{\lambda_{(2)}^{v}}{\mu_{(2)}\left(\tau_{(2)}\right.}\left(\mathbf{M}_{(2)}: \tilde{\mathbf{M}}\right)
$$


CTE of single

ASMEC lamina along the fiber direction

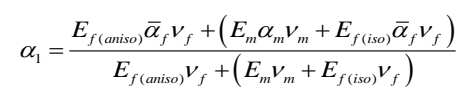

Transverse to the fiber direction

$\alpha_{2}=\left(1+V_{f(\text { taniso })}\right) \bar{\alpha}_{f} V_{f}+\left(1+V_{m}\right) \alpha_{m} V_{m}+$

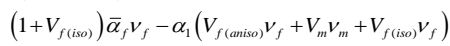

\subsection{Material parameter identification}

The present thermomechanical model has a total of 24 material parameters. Among them, two are associated with the glass transition temperature and can be obtained from the Differential Scanning Calorimetry (DSC) tests in Section 2.1; three parameters for nonlinear thermal expansion are identified by the CTE experiments on the PVAc fiber and single layer ASMEC; 15 parameters associated with the isotropic and anisotropic parts of the fibrous mat and the matrix can be identified from analysis of stress-strain curves of the fibrous mat and the single ASMEC lamina at different fiber directions; the remaining 4 parameters related to the temperature shift characteristics of the fiber can be identified by the temperature-strain curves in the third heating step of the representative shape memory cycle, as presented in section 2.3 . In this section, we show the results of the major steps in parameter identification. More details about each step can be found in Section 4 of Supplementary Materials. The final values of these material parameters were obtained by fitting the curves from the experiments at the programming temperature (or room temperature) and are listed in Table 4.

Table.4 Material parameters used in simulations

\begin{tabular}{lcc}
\hline Description & parameter & Value \\
\hline Matrix & & \\
Crosslinking density & $n\left(\mathrm{~m}^{-3}\right)$ & $1.96 \times 10^{26}$ \\
Fiber mat & $D(\mathrm{MPa})$ & 490 \\
Bulk modulus & $V_{F}$ & 0.32 \\
Fiber fraction & $\mu_{F(\text { iso })}(\mathrm{MPa})$ & 9.8 \\
Shear modulus for isotropic part & $\mu_{F(\text { aniso })}(\mathrm{MPa})$ & 46.2 \\
Shear modulus for anisotropic part & 34 &
\end{tabular}


Material index for anisotropic part

c

$-0.064$

Relaxation time

Reduced modulus index

$\tau_{0(1)}, \tau_{0(2)}(\mathrm{s})$

$9 \times 10^{9}$

\section{Yield parameter}

Initial shear strength for isotropic part

$s_{(1)}(\mathrm{MPa})$

Initial shear strength for anisotropic part

$s_{(2)}(\mathrm{MPa})$

Saturation shear strength for isotropic part

$s_{s(1)}(\mathrm{MPa})$

Saturation shear strength for anisotropic part

$s_{s(2)}(\mathrm{MPa})$

Zero stress level activation energy

$$
\Delta G(J)
$$

$5 \times 10^{-20}$

Prefactor parameter

$h_{o}(\mathrm{MPa})$

400

\section{Thermal component and time-temperature shifting parameters}

WLF reference temperature

WLF constant

WLF constant

Pre-exponential Arrhenius factor

Glass transition temperature of PVAc fiber

Glass transition temperature of Sylgard matrix

Linear rubbery coefficient of thermal expansion

Linear glassy coefficient of thermal expansion

Linear coefficient of Sylgard

$\begin{array}{cc}T_{M}\left({ }^{\circ} \mathrm{C}\right) & 30 \\ C_{1}(-) & 17.44\end{array}$

$C_{2}\left({ }^{\circ} \mathrm{C}\right) \quad 51.6$

$A F_{c} k_{B}^{-1}(\mathrm{~K}) \quad-25500$

$T_{g}\left({ }^{\circ} \mathrm{C}\right) \quad 42$

$T_{g}\left({ }^{\circ} \mathrm{C}\right) \quad-114$

$\alpha_{r}\left(1 /{ }^{\circ} \mathrm{C}\right) \quad 2.21 \times 10^{-4}$

$\alpha_{g}\left(1 /{ }^{\circ} \mathrm{C}\right) \quad 1.02 \times 10^{-4}$

$\alpha_{m}\left(1 /{ }^{\circ} \mathrm{C}\right) \quad 3.1 \times 10^{-4}$

\subsubsection{Uniaxial tension of the fibrous mat and single ASMEC lamina}

For uniaxial tension tests, we prescribe the displacement in the stretching direction (x-direction). According to the experiments, the traction free boundary conditions on the two lateral directions need to be satisfied, i.e. $\sigma_{2}=\sigma_{3}=0$. We then solve the constitutive relations for a given principal stretch. The detailed process is described in Section 5 of the Supplementary Material.

Fig. 10a shows the results of fitting and simulated stress-strain curves for PVAc fibrous mats of different angles with experimental results. Here, the tensile moduli and the yield stresses for 
the isotropic and anisotropic parts of the fibrous mat are separately determined by uniaxial stretch experiments along $\left(\theta=0^{\circ}\right)$ and transverse to the fiber direction $\left(\theta=90^{\circ}\right)$. From Fig. 10a, we can see very good agreement between the model simulations and experiments for the two fitting cases of $\theta=0^{\circ}$ and $\theta=90^{\circ}$. The model also captures the three intermediate cases of $\theta=$ $22.5^{\circ}, \theta=67.5^{\circ}$ and $\theta=45^{\circ}$.

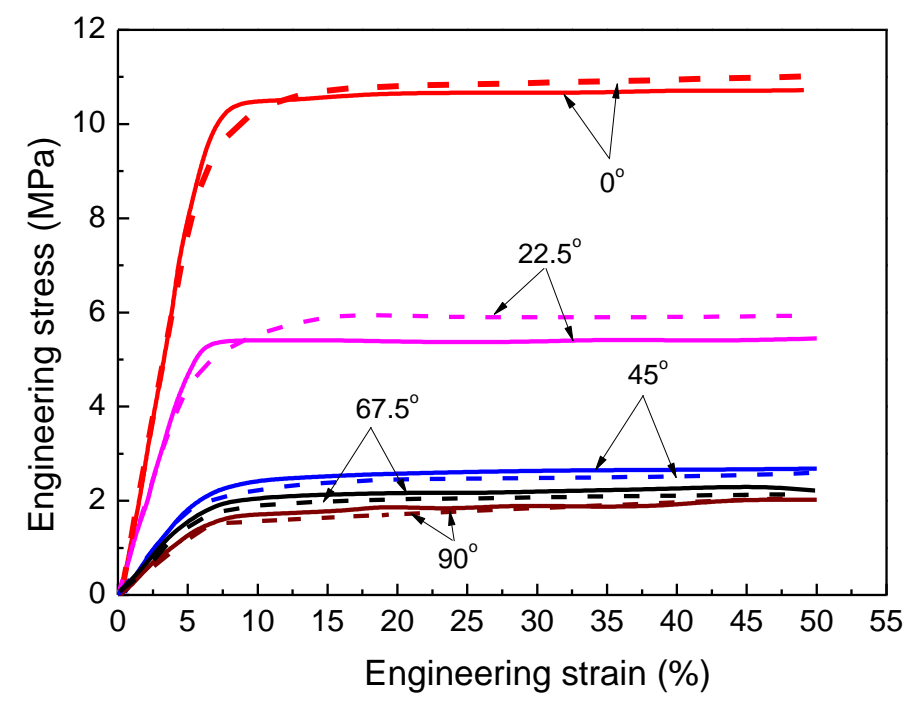

(a)

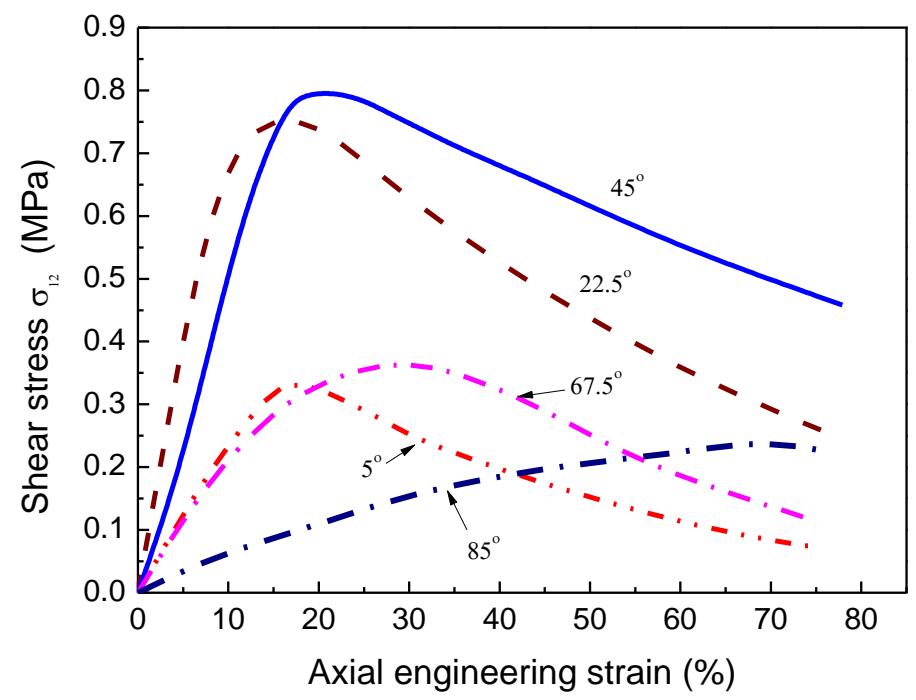

(b) 
Figure 10. (a) Engineering stress-engineering strain and (b) shear stress-axis engineering strain for PVAc fibrous mat as a function of fiber orientation angles of $0^{\circ}, 22.5^{\circ}, 45^{\circ}, 67.5^{\circ}, 90^{\circ}$. In (a), experimental results are denoted as solid lines and simulation results are dash dot lines.

For anisotropic materials, uniaxial stretching leads to non-zero shear stress. Fig. 10b shows the simulated shear stress, $\sigma_{12}$, as a function of stretch for different fiber orientations. Here the cases of $5^{\circ}$ and $85^{\circ}$ instead of $0^{\circ}$ and $90^{\circ}$ are plotted since there would be no shear force when fiber directions are $0^{\circ}$ and $90^{\circ}$. Overall, the shear stress increases with stretch then decreases after passing the yielding point. Further, the highest shear stress occurs when the fiber orientation is $\theta=45^{\circ}$.

\subsubsection{Single ASMEC lamina}

The material parameters for the matrix are obtained through uniaxial tension experiments on the single ASMEC lamina. In fitting the curves for the lamina, the material parameters associated with the fibrous mat are those obtained from fitting the fibrous mat curves. However, in simulating the mechanical behavior of the single ASMEC lamina, we find that the fibrous mat shows a modulus reduction after being infiltrated with Sylgard. As shown in the fabrication process(Rodriguez et al., 2013), the fibrous mat underwent a great dilation and the fibrous mat thickness increased approximately threefold after infiltration with Sylgard. Consequently, the fiber volume fraction decreased and the bonding strength between fibers in the single ASMEC lamina was reduced compared to that of the fibrous mat state. Therefore, we assume that the integrity of the fibrous mat was "damaged". To reflect this damage, we introduce a damage value, $\omega$, of 0.73 . The simulation results are compared to the experiment results in Fig.11. It can be found from the figure that the simulations on uniaxial stretch of single ASMEC lamina have a reasonable agreement with the experimental results. 


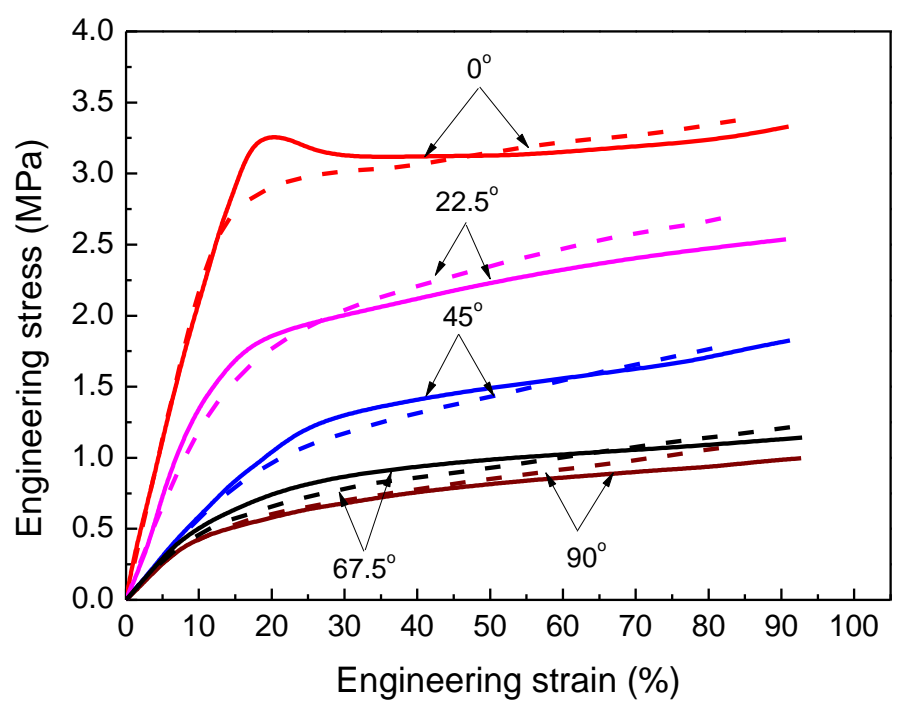

Figure11. Engineering stress-engineering strain for single ASMEC lamina as a function of fiber orientation angles of $0^{\circ}, 22.5^{\circ}, 45^{\circ}, 67.5^{\circ}, 90^{\circ}$. Experimental results are denoted as solid lines, and simulation results are dashed lines.

\subsection{Cold programmed shape memory behaviors}

\subsubsection{Cold programmed shape memory behavior of the single ASMEC lamina}

With the established constitutive model and fitted material parameters for single ASMEC lamina, the cold programmed shape memory behaviors are predicted by simulations and compared with the experimental results (shown in Section 2). For the simulations, thermomechanical loading conditions follow the experimental process, as described previously in Section 2.3. 


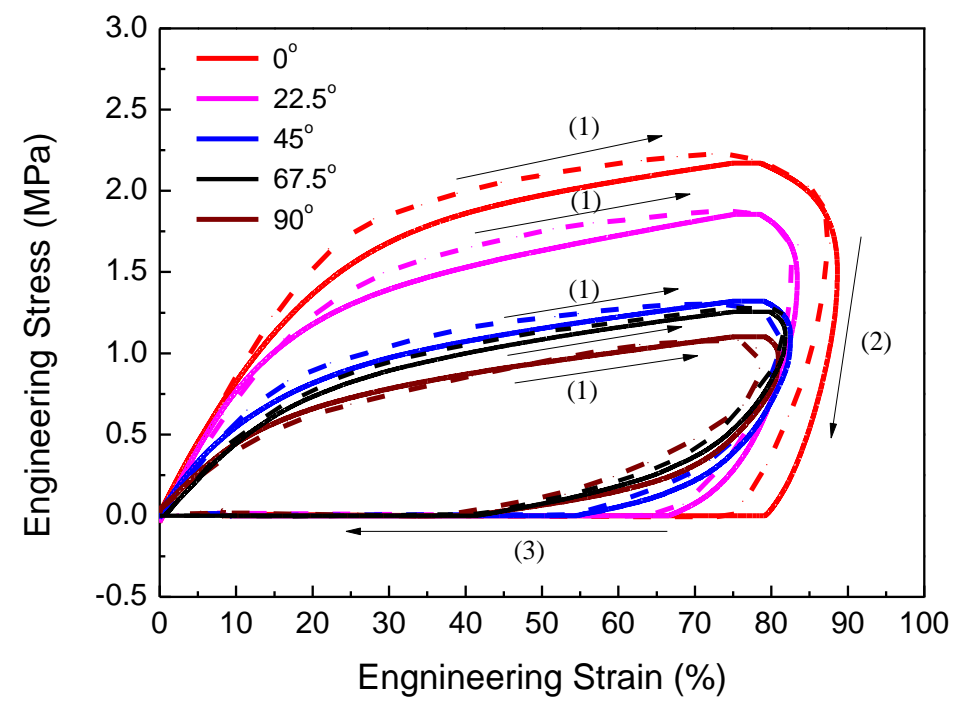

(a)

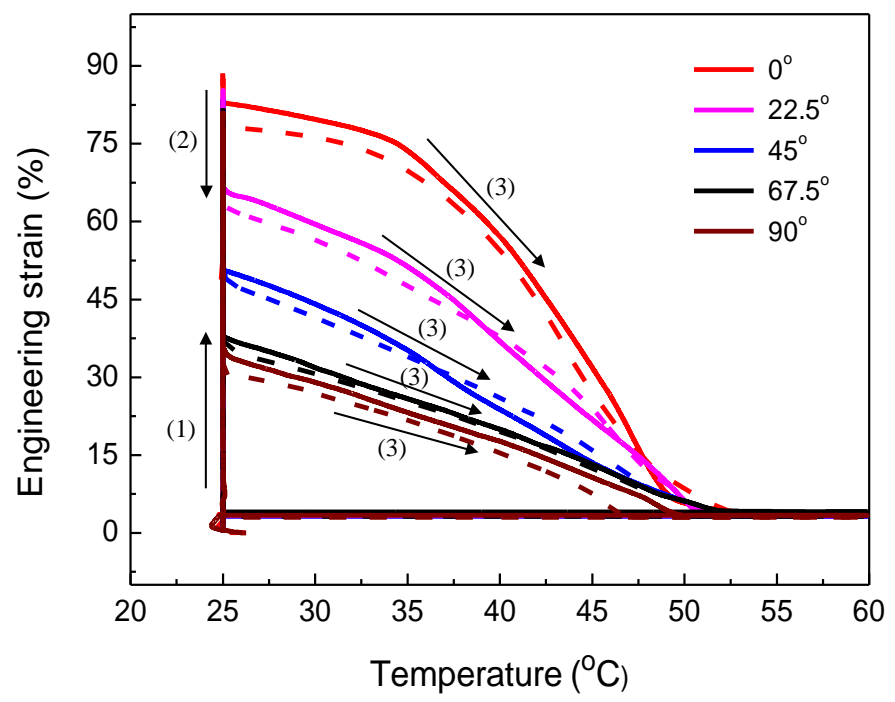

(b) 


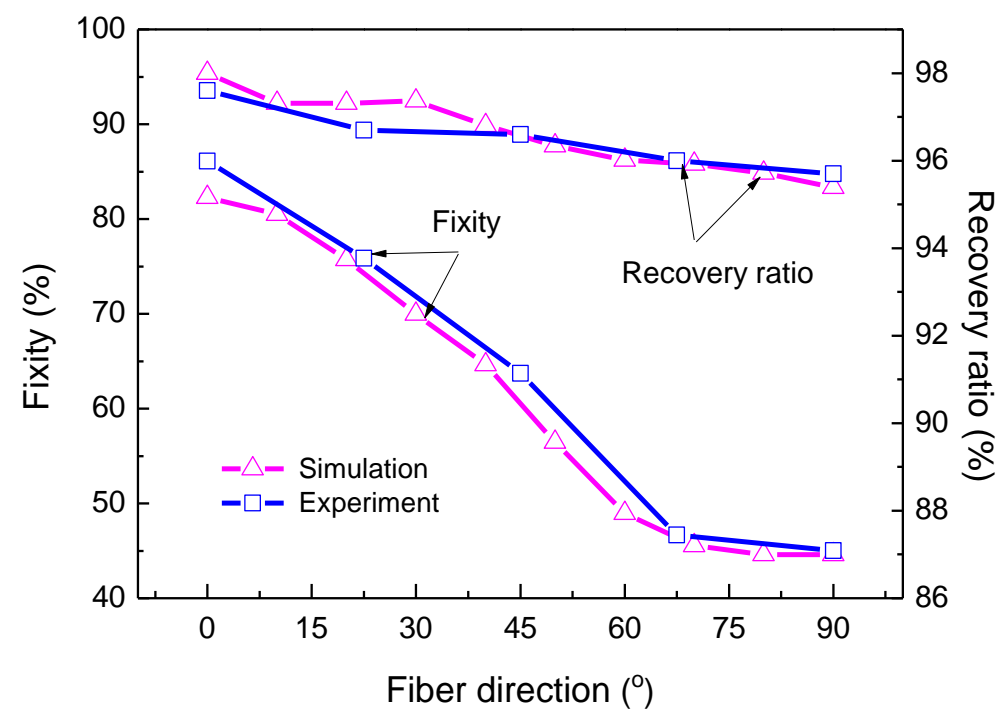

(c)

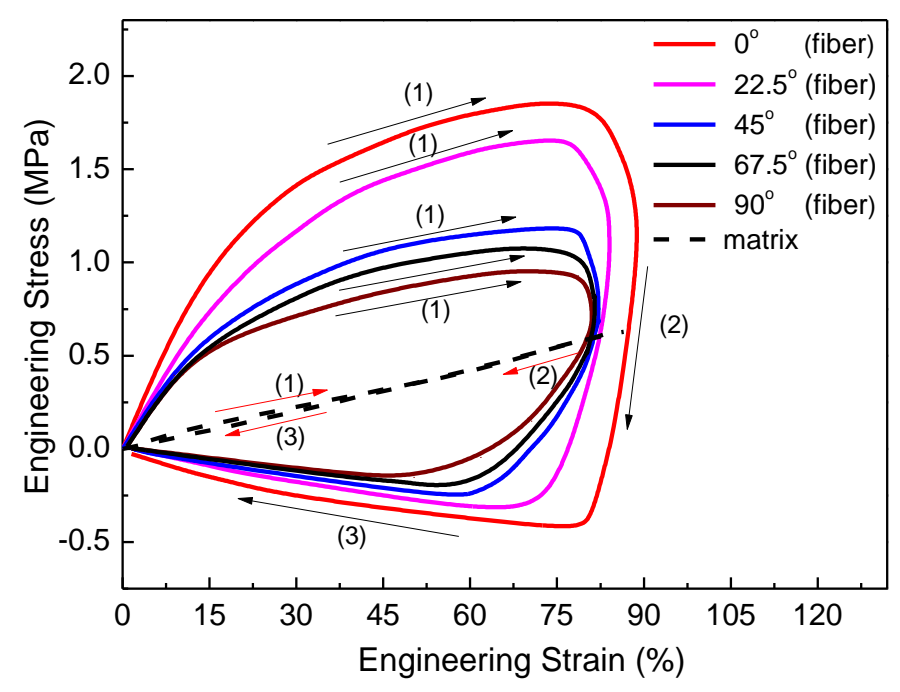

(d)

Fig.12 Cold programmed shape memory behaviors of single ASMEC lamina with different fiber orientation angles. (a) Engineering stress-engineering strain; (b) temperature-strain relation; (c) fixity and recovery ratios; (d) stress of fiber mat and matrix (solid lines indicate fiber stress, dashed line indicate stress of matrix). In (a) and (b), solid lines indicate experimental results and dashed lines indicate simulations. The shape recovery cycles consist of (1) loading at room temperature, (2) unloading at room temperature for fixing, and (3) heating to $60{ }^{\circ} \mathrm{C}$ for recovery.

The simulated cold programmed shape memory cycle of single ASMEC lamina for different 40 
fiber directions and the comparison with experiments are presented in Fig.12. It should be noted that the experiments used load control, which is different from the displacement-controlled experiments for material parameter identification. Fig. 12a and b show adequate agreement between model predictions and experiments. In particular, the model shows excellent prediction for the cases of fiber orientations of $\theta=45^{\circ}, \theta=67.5^{\circ}$ and $\theta=90^{\circ}$ and good agreement for the cases of $\theta=0^{\circ}$ and $\theta=22.5^{\circ}$. Fig.12c shows the comparison of the fixity from simulations and experiments as functions of fiber orientation. From the figure, the fixity as a function of $\theta$ follows the behavior of the tensile modulus, which highly depends on the anisotropic fiber part. The fixity decreases from $85 \%$ to $60 \%$ as $\theta$ increases, which is mainly due to the decrease in tensile modulus and yield stress when the fiber angle increases. When the fiber direction is greater than around $70^{\circ}$, only very small changes in fixity are expected as the fiber direction changes. Under this condition, resistance to recovery mainly comes from the viscoplastic deformation of the isotropic fiber part. It should be noted that in the experiments, a slight decrease in fixity could be observed over time; for the ASMEC laminates, a slight increase in radius could be observed over time. However, the changes were relatively small. Therefore, in this work the fixity is calculated immediately after programming process, and no delay is considered. Fig.12d presents the simulated stresses in the fiber mat (colored solid line) and in the matrix (black dashed line) in the ASMEC. Stresses in the fiber mat and matrix increase as the strain increases during the loading step at low temperature. During the unloading step, stresses in both the fibrous mat and the matrix decrease, but stresses in the fibrous mat transform into the compressive state as the matrix recovery surpasses the recovery of the plastically deformed fibers. In other words, the plastic strain in the fiber mat provides resistance to the recovery of the matrix. When the fiber angle is smaller (more aligned with the direction of deformation), a larger compression stress is observed, which leads to a higher fixity. No great effect of fiber direction on the recovery ratio is observed in the presently studied thermomechanical cycle.

\subsubsection{Parametric studies}

(a) Effect of loading rate 
Since the fibers are considered as a time-dependent material, we study the effects of loading rates on the SM performance. In the current simulations, the material is stretched to $80 \%$ strain using a controlled force mode with different loading rates: $0.0027 \mathrm{MPa} / \mathrm{min}, 0.027 \mathrm{MPa} / \mathrm{min}$, $0.13 \mathrm{MPa} / \mathrm{min}$ and $0.67 \mathrm{MPa} / \mathrm{min}$ (engineering stress). The same thermomechanical loading-unloading conditions for step 2 to step 4, described in Section 4.2.1, are used. Fig. 13a shows the effect of loading rate on the fixity for the single ASMEC lamina with different fiber angles. A high loading rate leads to high shape fixity. This is opposite to typical SM effects in amorphous shape memory polymers, where a slower loading rate normally leads to a higher fixity(Yu et al., 2014). This is because in an amorphous SMP, the development of viscous strain is the dominating factor for shape fixity, whereas for the ASMEC, the plastic strain in the fiber is the dominant factor.

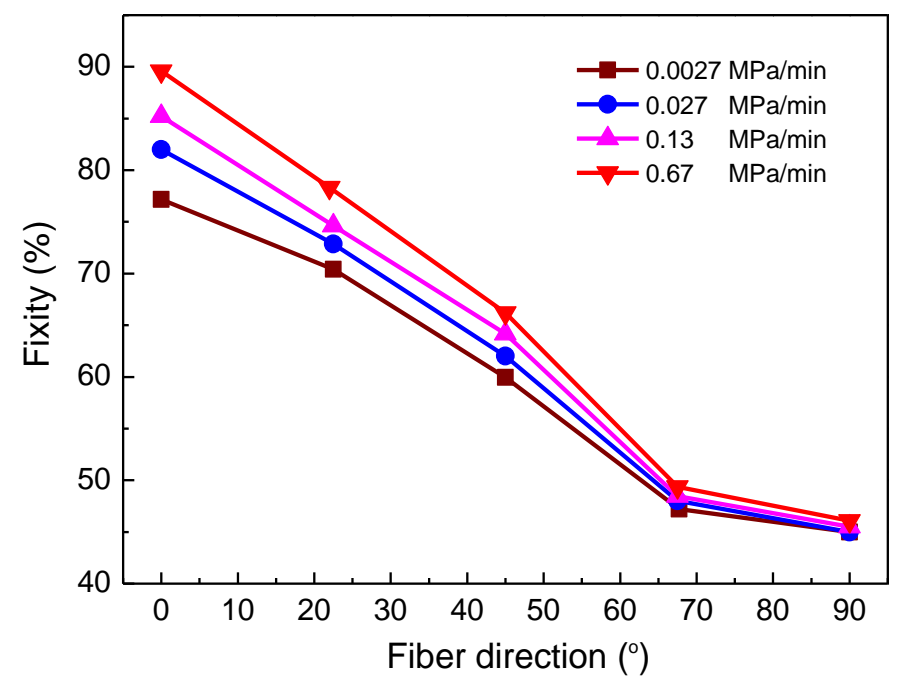

(a) 


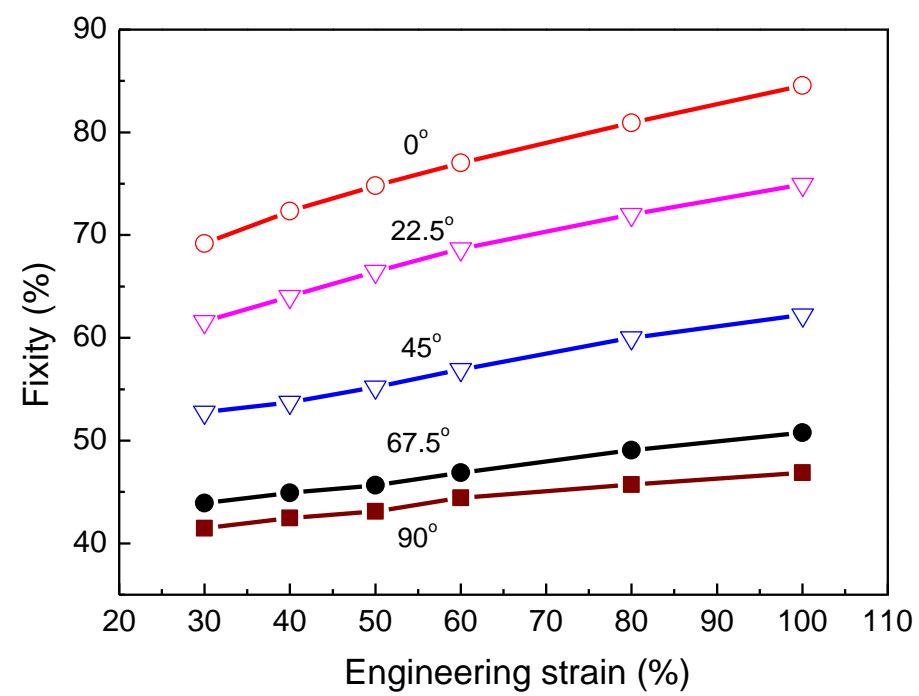

(b)

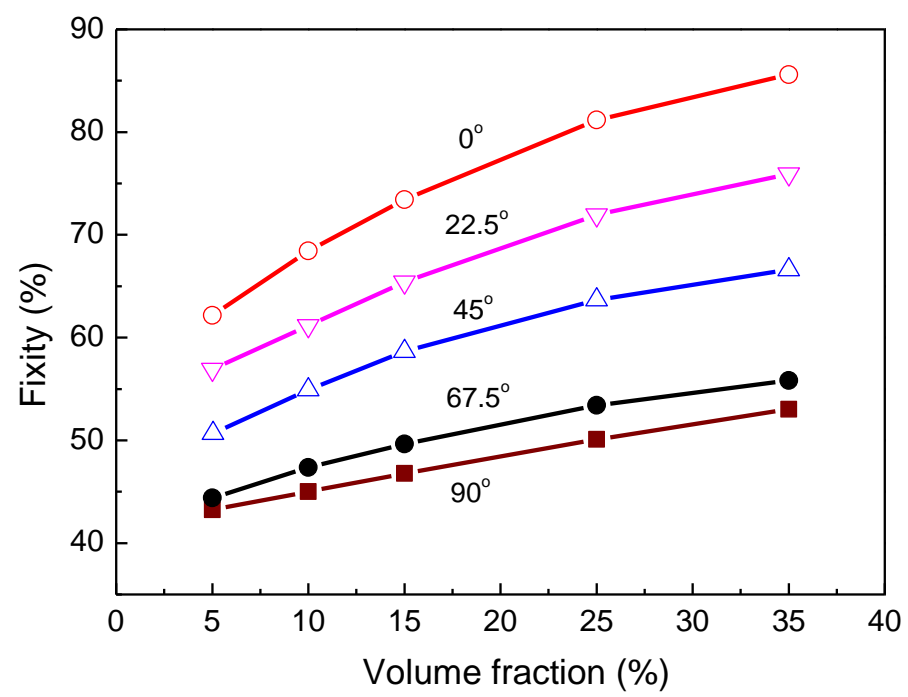

(c)

Figure 13. Shape fixity as a function of (a) fiber orientations and stress loading rates; (b) stretch strain; (c)fiber volume fraction.

(b) Effect of strain on shape fixity

Fig. 13b shows the effect of strain on the shape fixity. Here, the same thermomechanical loading-unloading conditions were used as those in the above section, except the samples were 
stretched to a range of strains spanning 30 to $100 \%$ using the controlled force mode at a rate of $0.0267 \mathrm{MPa} / \mathrm{min}$. For any specified fiber angle, a larger strain normally induces greater shape fixity. This is because a larger strain leads to a larger plastic deformation, which has a positive effect on the fixity. It is noted that the shape fixity is more sensitive to the strain when the fibers are aligned to the stretch direction. For example, when the loading direction is along the fiber direction, i.e., $\theta=0^{\circ}$, an increase of $21.3 \%$ in the shape fixity is obtained when the stretch strain is increased from $30 \%$ to $100 \%$; at $\theta=90^{\circ}$, we see an increase of about $11.3 \%$ in the shape fixity. This is because the fibers have more plastic strain when stretched along the fiber directions.

(c) Effect of volume fraction on shape fixity

Now, we turn to the effect of the fiber (PVAc) volume fraction on the fixity of the single ASMEC lamina. All of the material parameters and thermomechanical conditions are set as those in Section 2.2.1. The simulated fixity are plotted in Fig.13c for single ASMEC lamina with fiber volume fractions varying from $5 \%$ to $35 \%$. Generally, fixing capability directly depends on the fiber's ability to resist the contraction of the Sylgard matrix. From the figure, an increase in fiber volume fraction leads to an obvious increase of the fixity. This effect is more obvious when the angle between the fiber and load directions is comparatively small, where the fibrous mat has a dominant effect over the rubbery matrix.

\subsection{ASMEC Laminate}

\subsubsection{Model for ASMEC laminate}

Here, we show that the developed constitutive model can be applied to study the cold programming and curling effects of the ASMEC laminate. For the purpose of simplicity, we consider the system as a bilayer model by neglecting the adhering layer. The theoretical model for the laminated ASMECs is established by applying the Euler- Bernoulli beam theory together with the anisotropic viscoplastic model for the single ASMEC lamina.

To model the cold-programmed induced curling, three variables, in addition to the stretch variables $\left(\lambda_{1} \lambda_{2} \lambda_{3}\right)$, are introduced: radius of curvature $(R)$, in-plane rotation angle $(\varphi)$, and the 
pitch $\left(H_{\text {pitch }}\right)$, as shown in Fig. 14a. Here, the shear angle is the result of displacement controlled loading, where the strip is kept flat and a large shear force emerges in the bilayer system. When unclamped, the shear force is released, and the difference in recovery of the viscoplastic fibrous mat and the hyperelastic elastomer gives rise to a shear deformation, $\theta$, in the laminated system. The pitch, which is the distance between two consecutive peaks of the curled sample (as shown in Fig.14a), can be calculated as

$$
H_{\text {pitch }}=2 \pi R \tan \varphi
$$

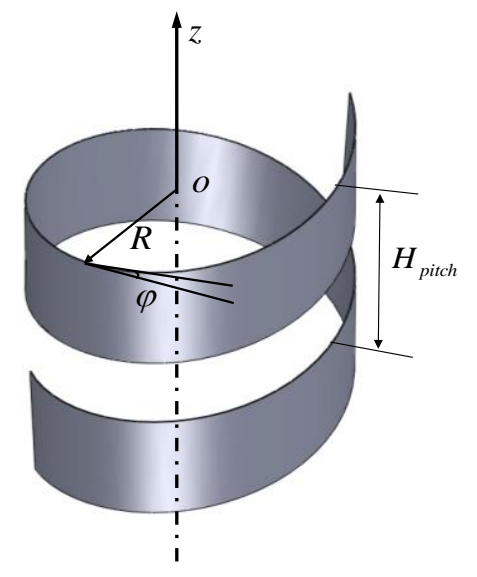

(a)

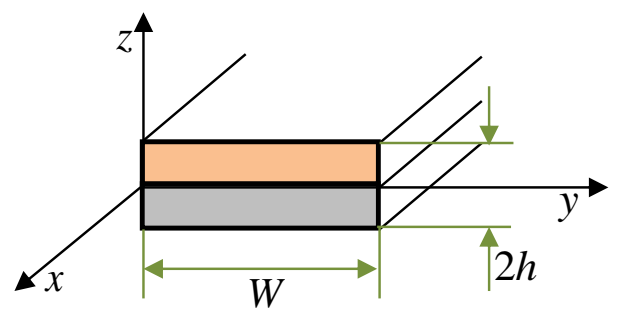

(b)

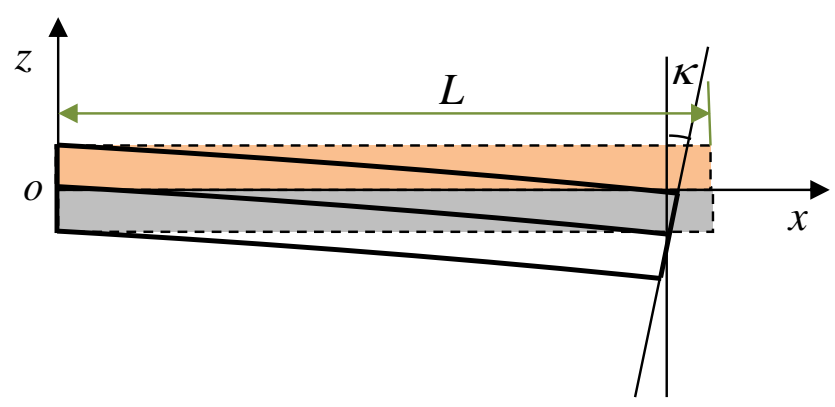

(c)

Figure 14. Schematic diagrams for (a) the helices curled configuration; and for the laminated ASMEC strip: (b) dimensions of the cross section; (c) the schematic of bending of laminated ASMECs.

A schematic of a laminated ASMEC is shown in Fig.14b. The $x, y$ plane passes through the 
middle of the laminated strip, where the $z$-coordinate is along the thickness of the laminated strip. The thicknesses of the top bottom layers are $h_{1}=h_{2}=h$, respectively. Here, we first assume that the top and bottom layers have the same thickness; we will discuss the effects of layer thickness on the radius of curvature and pitch at the end. The ratio of length to thickness is $43(=30 \mathrm{~mm} / 0.7 \mathrm{~mm})$ for current analyzed strip. Therefore, for the purpose of simplicity, the Euler-Bernoulli beam theory is applied in simulating the curling property of strip by using current proposed cold programmed shape memory model. The analytical model developed is thus based on the assumption that higher order terms, such as transversal shear, are ignored. Taking the strain at $z=0$ as $\varepsilon_{o}\left(\varepsilon_{1}(0)=\varepsilon_{o}\right)$, the axial strain due to bending along the $z$-axis follows(Ge et al., 2014)

$$
\varepsilon_{1}(z)=\varepsilon_{0}+\kappa \cdot z
$$

where $\kappa(\kappa=1 / R)$ is the curvature. As the width is much smaller than the length, the curvature along the axial direction is rationally neglected, and the strain, $\varepsilon_{2}$, is considered constant along the thickness. Here the engineering strain increments, $\Delta \varepsilon_{i}$, are related to the stretch increment as $\Delta \varepsilon_{i}=\Delta \lambda_{i}-1(i=1,2,3)$. From the experimental procedures, two types of loading methods are applied, i.e., the displacement control in stretching the material, where bending and out of plane distortion is not allowed, and the force control in unloading, where the curled configuration appears in the forms of bending and twisting. Note that the displacement control is typical in the cold-programming step and the force control is used to calculate the unloaded shape change where the external force is zero. We separately present models for the displacement-loading and the force-unloading in the Section 7 of the Supplementary Materials, where detailed solving methods are provided for bilayer ASMECs.

\subsubsection{Laminate bending results}

The above discussed model is used to calculate the bending curvature of the laminates as functions of applied strain, as shown in Fig.15, for comparison to the experimental results. Overall, the model matches experiment very well. From the figure, it can be found that as stretch 
increases, a greater curvature is induced. In addition, an increase in fiber orientation leads to a larger curvature. The error is supposed to be induced by the neglect of effects the interface and the assumption of equal thicknesses in the bottom and top layers.

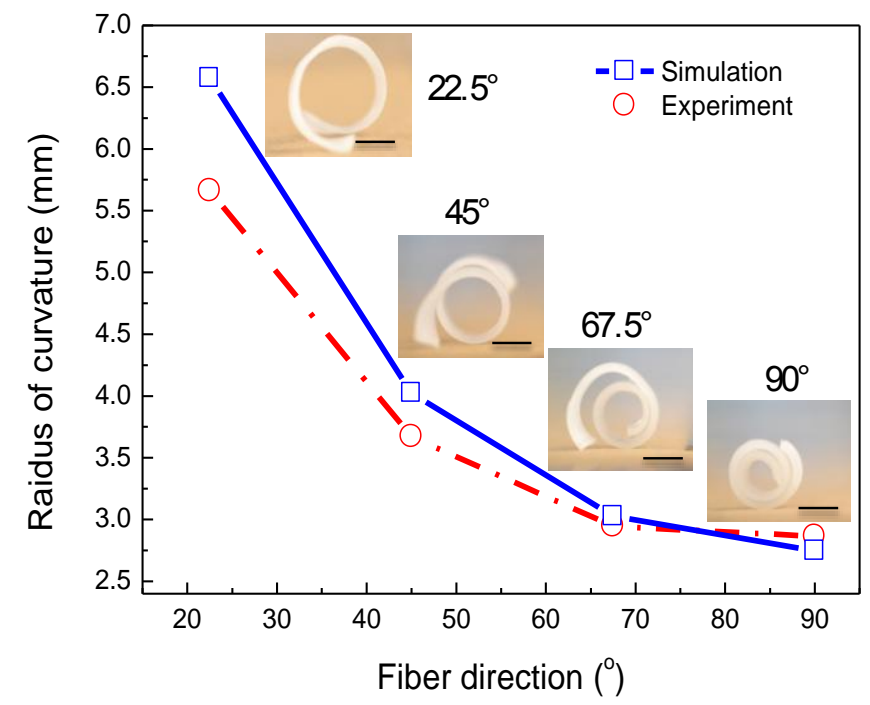

(a)

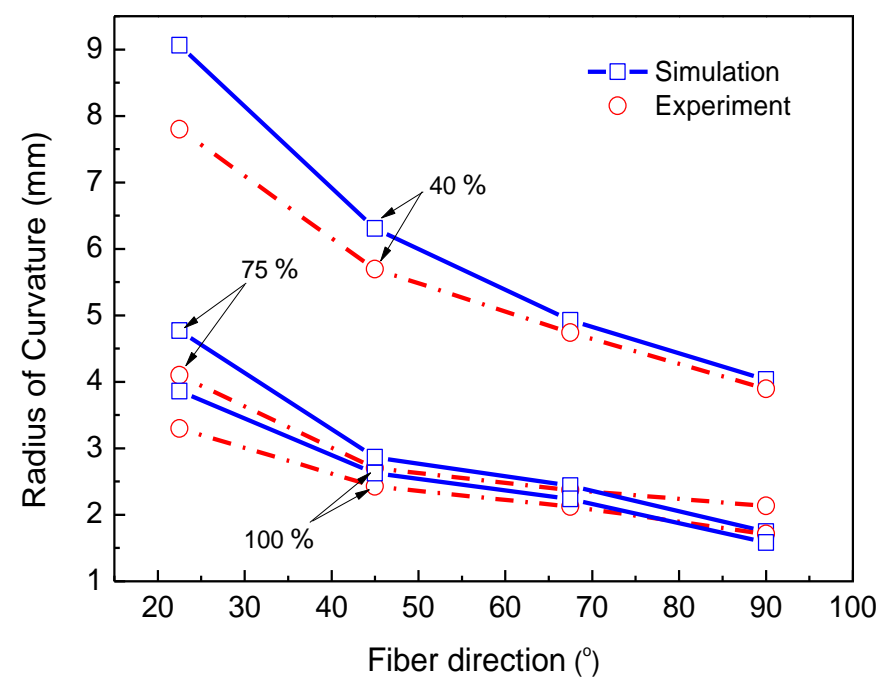

(b)

Figure 15. Simulated radius of curvature of the bilayer ASMEC as a function of fiber direction and compared with the experimental results for different strain, (a) $50 \%$, (b) $40 \%, 75 \%$ and $100 \%$. The red dashed lines with circles correspond to simulations, while the continuous blue 
lines with circles correspond to the experiment. In (a) the inset photos show the experimental results with the scale bars representing $4 \mathrm{~mm}$.

\subsubsection{Pitches}

We now turn our attention to the predictions of pitch for the curled laminated ASMECs as a function of fiber orientation, and a comparison is made with experimental measurements, as shown in Fig.16 a and b. Overall, the model predicts well the pitch as a function of fiber orientation. It is observed that pitch decreases with increasing fiber angle. This is because increasing the fiber angle will decrease the in-plane rotation and thus decrease the pitch. It is also found that pitch generally decreases with increasing strain, as shown in Fig. 16c. But when the strain increases to above $75 \%$, pitch becomes not so sensitive to the strain. This is because at the larger strains, although the in-plane rotation angle increases, the radius of curvature decreases; the lumped effect causes a plateau in the pitch-strain curve.

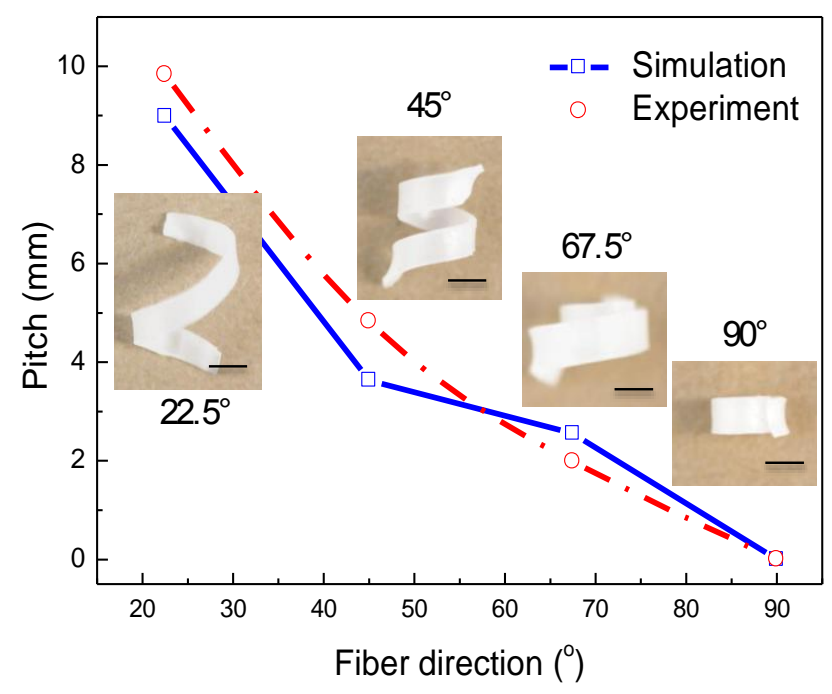

(a) 


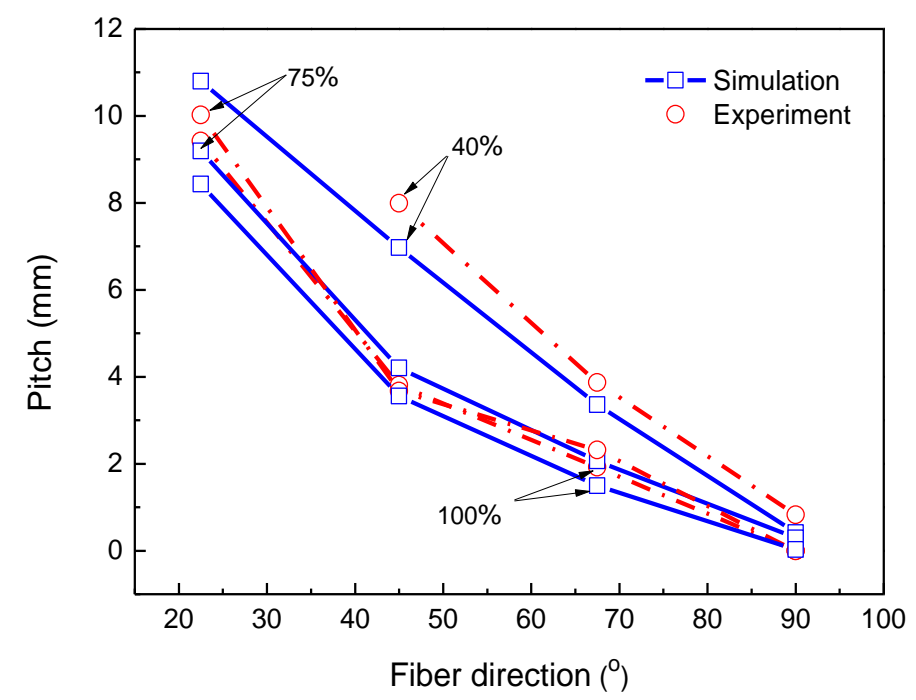

(b)

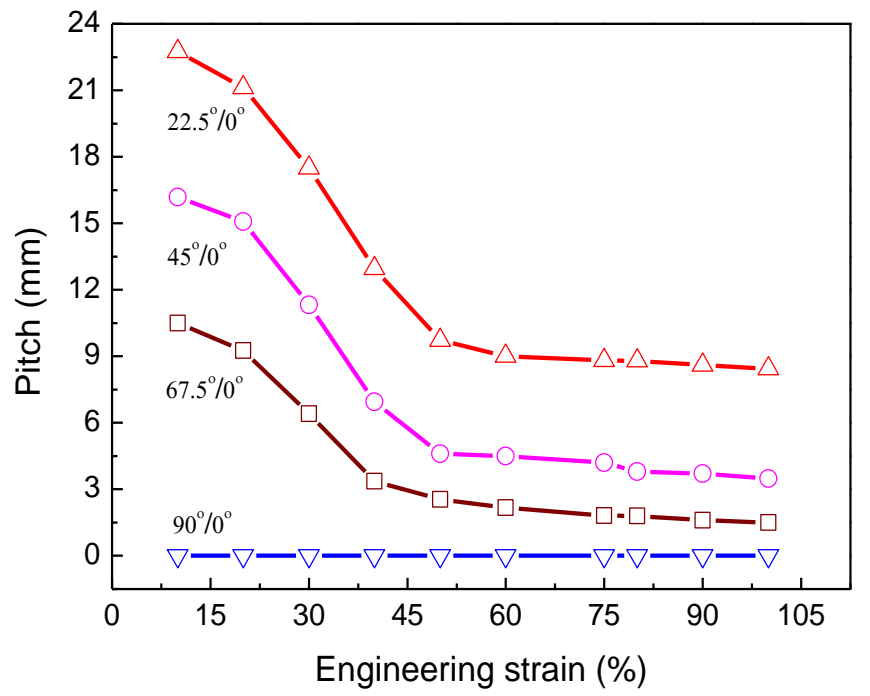

(c)

Figure 16. The simulated pitches of the bilayer ASMEC as a function of fiber direction and compared with the experimental results for different strain, (a) 50\%, (b) $40 \%, 75 \%$, and $100 \%$. (c) The simulated pitches of the bilayer ASMEC as a function of strain. In (a) the inset photos show the experimental results with the scale bars representing $4 \mathrm{~mm}$. 


\subsubsection{Effects of thickness on pitch and curvature radius}

In the above analysis, we assume equal thickness of the two layers. But, from the above discussions, the configuration of the laminated ASMEC results from a combination of twisting, compression and bending, together with the anisotropic viscous properties and dimensions of material. The effect of each layer's thickness on the curled configuration of the laminated ASMEC is discussed here. Fig. 17 presents the effect of thickness on pitch for the laminated ASMECs. Here the fiber orientation is considered as $\left(45^{\circ} / 0^{\circ}\right)$ and the same total thickness as the experiment is used as a reference. From the figure it can be found when the thickness of the top layer $\left(h_{1}\right)$ increases, the pitch increases. This is because as the top thickness increases, the anisotropic effect of the top layer becomes more dominant and the in-plane rotation angle becomes greater. The opposite is true when the thickness of the bottom layer is increased. Fig. 17 shows the additional design parameter that can be used for controlling the pitch.

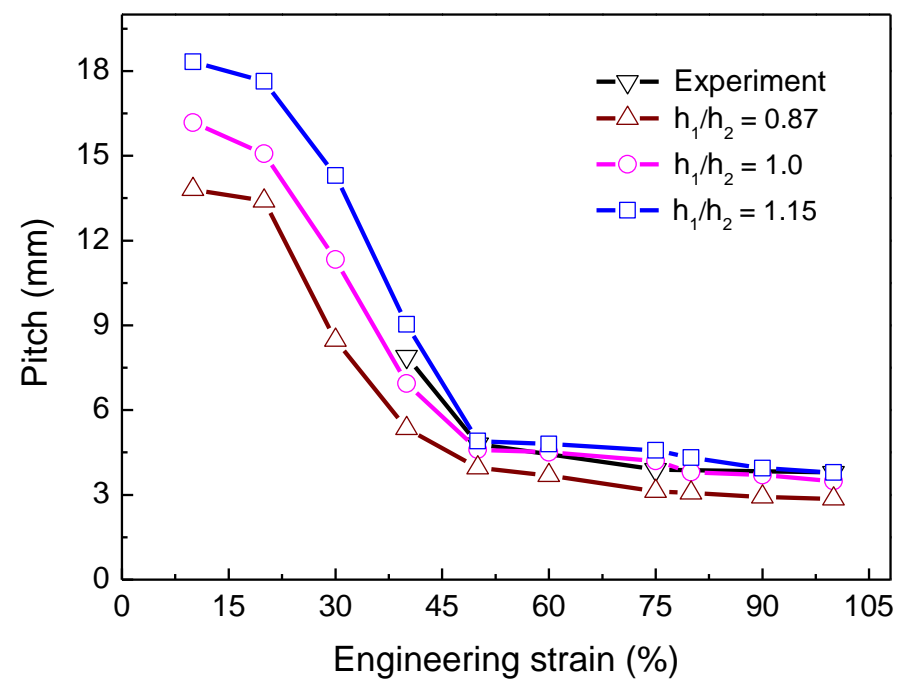

Figure 17. The pitch as a function of strain for ASMECS laminate $\left(45^{\circ} / 0^{\circ}\right)$ with different thickness ratios.

\section{Conclusions}

We studied the thermoviscoplastic behavior of anisotropic shape memory elastomeric composites (ASMECs), which consists of an elastomeric matrix reinforced by an aligned 
amorphous polymer fibrous network. The elastomeric matrix (Sylgard) provides entropic elasticity and the aligned fibrous mat shows viscoplastic behavior at low temperatures. A temporary shape can be achieved upon unloading when the ASMEC is stretched at low temperatures, as the induced plastic-like strain in the fibrous mat resists the contractile force of the elastomer matrix. When increasing the temperature above the glass transition temperature, the fiber material behaves like a melt, leading to the recovery of the ASMEC to its equilibrium and flat state. We carried out extensive experiments to characterize and understand the anisotropic viscoplastic and shape memory behavior of this ASMEC. We also developed a 3D finite-deformation constitutive model to describe the complicated thermoviscoplastic behavior. In the model, the fiber mat is split into an isotropic part and an anisotropic part, and the 3D inelastic behavior of each part is captured by a nonlinear thermoviscoplastic model. The model adequately captures the anisotropic inelastic behavior of the fibrous mat and single ASMEC lamina, and provides insight into the anisotropic yielding property. We also used the model to explore the effect of fiber volume fraction, loading rate and stretch on shape fixity of the mechanically programmed shape memory behavior of ASMECs. These parametric studies show that high loading rates, larger stretch ratio, and high volume fraction of fibrous mat generally can provide larger fixity. The model also shows high accuracy in prediction of the cold-programmed curling of laminated ASMECs. From simulation, the curling configuration of laminated ASMECs highly depends on fiber direction, the stretched strain and the thickness of the layers. These parameters provide a broad design space for potential applications of ASMECs.

Acknowledgement: The authors acknowledge the financial support from NSF grants (CMMI-1441375, CMMI-1334658). The authors are grateful for helpful discussions with and some experimental works (SEM and some DMA tests) by Dr. Amir Torbati.

\section{Reference}

Adolf, D.B., Chambers, R.S., Caruthers, J.M., 2004. Extensive validation of a 
thermodynamically consistent, nonlinear viscoelastic model for glassy polymers. Polymer 45, 4599-4621.

Arruda, E.M., Boyce, M.C., 1993. Evolution of Plastic Anisotropy in Amorphous Polymers during Finite Straining. International Journal of Plasticity 9, 697-720.

Arzoumanidis, G.A., Liechti, K.M., 2003. Linear viscoelastic property measurement and its significance for some nonlinear viscoelasticity models. Mechanics of Time-Dependent Materials 7, 209-250.

Bergstrom, J.S., Boyce, M.C., 1998. Constitutive modeling of the large strain time-dependent behavior of elastomers. Journal of the Mechanics and Physics of Solids 46, 931-954.

Boyce, M.C., Parks, D.M., Argon, A.S., 1988. Large Inelastic Deformation of Glassy-Polymers .1. Rate Dependent Constitutive Model. Mechanics of Materials 7, 15-33.

DiMarzio, E.A., Yang, A.J.M., 1997. Configurational entropy approach to the kinetics of glasses. Journal of Research of the National Institute of Standards and Technology 102, 135-157.

Dupaix, R.B., Boyce, M.C., 2007. Constitutive modeling of the finite strain behavior of amorphous polymers in and above the glass transition. Mechanics of Materials 39, 39-52.

Ehret, A.E., Bol, M., Itskov, M., 2011. A continuum constitutive model for the active behaviour of skeletal muscle. Journal of the Mechanics and Physics of Solids 59, 625-636.

Ferry, J.D., 1980. Viscoelastic properties of polymers, 3d ed. Wiley, New York.

Ge, Q., Dunn, C.K., Qi, H.J., Dunn, M.L., 2014. Active origami by 4D printing. smart materials and structures 23, 15 .

Ge, Q., Luo, X.F., Rodriguez, E.D., Zhang, X., Mather, P.T., Dunn, M.L., Qi, H.J., 2012. Thermomechanical behavior of shape memory elastomeric composites. Journal of the Mechanics and Physics of Solids 60, 67-83.

Ge, Q., Qi, H.J., Dunn, M.L., 2013a. Active materials by four-dimension printing. Applied physics letters 103.

Ge, Q., Yu, K., Dunn, M.L., Qi, H.J., 2013b. Shape Memory Polymers: Mechanisms and Constitutive Models. International Journal of Aerospace and Lightweight Structures 3, 1-53.

Guo, Z.Y., Peng, X.Q., Moran, B., 2006. A composites-based hyperelastic constitutive model for soft tissue with application to the human annulus fibrosus. Journal of the Mechanics and Physics of Solids 54, 1952-1971.

Guo, Z.Y., Peng, X.Q., Moran, B., 2007. Mechanical response of neo-Hookean fiber reinforced incompressible nonlinearly elastic solids. International Journal of Solids and Structures 44, 1949-1969.

Holzapfel, G.A., Gasser, T.C., 2001. A viscoelastic model for fiber-reinforced composites at finite strains: Continuum basis, computational aspects and applications. Gomput. Methods Appl. Mech. Engrg. 190, 4379-4403.

Holzapfel, G.A., Gasser, T.C., Ogden, R.W., 2000. A new constitutive framework for arterial wall mechanics and a comparative study of material models. J Elasticity 61, 1-48.

Hu, J., Zhuo, H., 2010. Shape memory polymers in coatings and laminates for textiles. Woodhead Publ Text, 222-235.

Hu, J.L., Zhu, Y., Huang, H.H., Lu, J., 2012. Recent advances in shape-memory polymers: 
Structure, mechanism, functionality, modeling and applications. Prog Polym Sci 37, 1720-1763. Itskov, M., Ehret, A.E., Mavrilas, D., 2006. A polycovex anisotropic strain-energy function for soft collagenous tissues. Biomech Model Mechanbiol 5, 17-26.

Kao, P., 2011. Biaxial mechanical characterization and microstructure-driven modeling of elastic pulmonary artery walls of large mammals under hypertensive conditions.

Kao, P.H., Lammers, S.R., Tian, L., Hunter, K., Stenmark, K.R., Shandas, R., Qi, H.J., 2011. A Microstructurally Driven Model for Pulmonary Artery Tissue. Journal of Biomechanical Engineering-Transactions of the Asme 133.

Kardeniz, Z.H., Kumlutas, D., 2007. A numerical study on the coefficients of thermal expansion of fiber reinforced composite materials. Composite Structures 78, 1-10.

Knauss, W.G., Emri, I., 1987. Volume Change and the Nonlinearly Thermoviscoelastic Constitution of Polymers. Polym Eng Sci 27, 86-100.

Knauss, W.G., Emri, I.J., 1981. Non-Linear Viscoelasticity Based on Free-Volume Consideration. Comput Struct 13, 123-128.

Kovacs, A.J., Aklonis, J.J., Hutchinson, J.M., Ramos, A.R., 1979. Isobaric Volume and Enthalpy Recovery of Glasses .2. Transparent Multi-Parameter Theory. J Polym Sci Pol Phys 17, 1097-1162.

Kovacs, A.J., Aklonis, J.J., Hutchinson, J.M., Ramos, A.R., 1996. Isobaric volume and enthalpy recovery of glasses .2. A transparent multiparameter theory. J Polym Sci Pol Phys 34, 2467-2532.

Kroon, M., 2010. A continuum mechanics framework and a constitutive model for remodelling of collagen gels and collagenous tissues. Journal of the Mechanics and Physics of Solids 58, 918-933.

Lendlein, A., Kelch, S., 2002. Shape-memory polymers. Angew Chem Int Edit 41, 2034-2057.

Leng, J.S., Lan, X., Liu, Y.J., Du, S.Y., 2011. Shape-memory polymers and their composites: Stimulus methods and applications. Prog Mater Sci 56, 1077-1135.

Li, G., Xu, W., 2011. Thermomechanical behavior of thermoset shape memory polymer programmed by cold-compression: Testing and constitutive modeling. Journal of the Mechanics and Physics of Solids 59, 1231-1250.

Li, G.Q., Shojaei, A., 2012. A viscoplastic theory of shape memory polymer fibres with application to self-healing materials. P Roy Soc a-Math Phy 468, 2319-2346.

Lion, A., 2000. Constitutive modelling in finite thermoviscoplasticity: a physical approach based on nonlinear rheologicla models. International Journal of Plasticity 16, 469-494.

Liu, C., Qin, H., Mather, P.T., 2007. Review of progress in shape-memory polymers. J. Mater. Chem. 17, 1543-1558.

Lu, H.B., Huang, W.M., Yao, Y.T., 2013. Review of chemo-responsive shape change/memory polymers. Pigment \& Resin Technology 42, 237-246.

Luo, X.F., Mather, P.T., 2009. Preparation and characterization of shape memory elastomeric composites. Macromolecules 42, 7251-7253.

Meng, H., Li, G.Q., 2013. A review of stimuli-responsive shape memory polymer composites. Polymer 54, 2199-2221. 
Milani, A.S., Nemes, J.A., 2004. An intelligent inverse method for characterization of textile reinforced thermoplastic composites using a hyperelastic constitutive model. Composites Science and Technology 64, 1565-1576.

Nedjar, B., 2007. An anisotropic viscoelastic fibre-matrix model at finite strains: Continuum formulation and computational aspects. Comput. Methods. Appl. Mech. Engrg. 196, 1745-1756.

Nguyen, T., Qi, H., Castro, F., Long, K., 2008a. A thermoviscoelastic model for amorphous shape memory polymers: Incorporating structural and stress relaxation. Journal of The Mechanics and Physics of Solids 56, 2792-2814.

Nguyen, T.D., 2013. Modeling Shape-Memory Behavior of Polymers. Polym Rev 53, 130-152.

Nguyen, T.D., Jones, R.E., Boyce, B.L., 2007. Modelling the anisotropic finite-deformation viscoelastic behavior of soft fiber-reinforced composites. International journal of solids and structures 44, 8366-8389.

Nguyen, T.D., Jones, R.E., Boyce, B.L., 2008b. A Nonlinear Anisotropic Viscoelastic Model for the Tensile Behavior of the Corneal Stroma. Journal of Biomechanical Engineering 130, 041020. O'Connell, P.A., McKenna, G.B., 1999. Arrhenius-type temperature dependence of the segmental relaxation below Tg. Journal of Chemical Physics 110, 11054-11060.

Peng, X.Q., Guo, Z.Y., Moran, B., 2006. An Anisotropic Hyperelastic Constitutive Model With Fiber-Matrix Shear Interaction for the Human Annulus Fibrosus. Journal of Applied Mechanics 73, 815.

Ping, P., Wang, W., Chen, X., Jing, X., 2005. Poly( $\varepsilon$-caprolactone) Polyurethane and Its Shape-Memory Property. Biomacromolecules 6, 587-592.

Popelar, C.F., Liechti, K.M., 2003. A distortion-modified free volume theory for nonlinear viscoelastic behavior. Mechanics of Time-Dependent Materials 7, 89-141.

Qi, H., Boyce, M., 2005. Stress-strain behavior of thermoplastic polyurethanes. Mechanics of Materials 37, 817-839.

Qi, H., Nguyen, T., Castro, F., Yakacki, C., Shandas, R., 2008. Finite deformation thermo-mechanical behavior of thermally induced shape memory polymers. Journal of Mechanics and Physics of Solids 56, 1730-1751.

Reese, S., Govindjee, S., 1998. A theory of finite viscoelasticity and numerical aspects. International Journal of Solids and Structures 35, 3455-3482.

Reinert, F., Luthy, W., 2005. Thermo-optically driven adaptive mirrr based on thermal expansion: preparation and resolution. Optics Express 13, 10750.

Robertson, J.M., Torbati, A.H., Rodriguez, E.D., Mao, Y.Q., Baker, R.M., Qi, H.J., Mather, P.T., 2015. Mechanically programmed shape change in laminated elastomeric composites. Soft Matter 11, 5754-5764.

Rodriguez, E.D., Weed, D.C., Mather, P.T., 2013. Anisotropic shape-memory elastomeric composites: fabrication and testing. Macromolecular Chemistry and Physics 214, 1247-1257.

Rosen, B.W., Hashin, Z., 1970. Effective thermal expansion coefficients and specific heats of composite materials. Int J Eng Sci 8, 157-173.

Schapery, R.A., 1968. Thermla expansion coefficients of composite materials based on energy principles. J. Compos Mater 2, 380-404. 
Shojaei, A., Li, G.Q., Voyiadjis, G.Z., 2013. Cyclic Viscoplastic-Viscodamage Analysis of Shape Memory Polymers Fibers With Application to Self-Healing Smart Materials. J Appl Mech-T Asme 80.

Siliberstein, M.N., Pai, C.-L., Rutledge, G.C., Boyce, M.C., 2012. Elastic-plastic behavior of non-woven fibrous mats. Journal of the Mechanics and Physics of Solids 60, 295-318.

Srivastava, V., Chester, S.A., Ames, N.M., Anand, L., 2010a. A thermo-mechanically-coupled large-deformation theory for amorphous polymers in a temperature range which spans their glass transition. International Journal of Plasticity 26, 1138-1182.

Srivastava, V., Chester, S.A., Anand, L., 2010b. Thermally actuated shape-memory polymers: Experiments, theory, and numerical simulations. Journal of the Mechanics and Physics of Solids $58,1100-1124$.

Sun, D.D.N., Leong, K.W., 2004. A Nonlinear Hyperelastic Mixture Theory Model for Anisotropy, Transport, and Swelling of Annulus Fibrosus. Annals of Biomedical Engineering 32, 92-102.

Tool, A.Q., 1946. Relation between inelastic deformability and thermal expansion of glass in its annealing range. Journal of the American Ceramic Society 29, 240-253.

Tool, A.Q., 1948. Effect of heat-treatment on the density and constituttion of high-silica glasses of the borosilicate type. Journal of the American Ceramic Society 31, 177-186.

Westbrook, K.K., Kao, P.H., Castro, F., Ding, Y., Jerry Qi, H., 2011. A 3D finite deformation constitutive model for amorphous shape memory polymers: A multi-branch modeling approach for nonequilibrium relaxation processes. Mechanics of Materials 43, 853-869.

Williams, M.L., Landel, R.F., Ferry, J.D., 1955. Temperature Dependence of Relaxation Mechanisms in Amorphous Polymers and Other Glass-Forming Liquids. Physical Review 98, 1549-1549.

Yang, W.G., Lu, H.B., Huang, W.M., Qi, H.J., Wu, X.L., Sun, K.Y., 2014. Advanced Shape Memory Technology to Reshape Product Design, Manufacturing and Recycling. Polymer 6, 2287-2308.

Yu, K., Ge, Q., Qi, H.J., 2014. Reduced time as a unified parameter determining fixity and free recovery of shape memory polymers. Nat Commun 5, 3066. 